

\title{
Preperiodic points for families of polynomials
}

\author{
Dragos Ghioca, Liang-Chung Hsia and Thomas J. Tucker
}

Let $a(\lambda), b(\lambda) \in \mathbb{C}[\lambda]$, and let $f_{\lambda}(x) \in \mathbb{C}[x]$ be a one-parameter family of polynomials indexed by all $\lambda \in \mathbb{C}$. We study whether there exist infinitely many $\lambda \in \mathbb{C}$ such that both $a(\lambda)$ and $b(\lambda)$ are preperiodic for $f_{\lambda}$.

\section{Introduction}

The classical Manin-Mumford conjecture for abelian varieties (now a theorem due to Raynaud [1983a; 1983b]) predicts that the set of torsion points of an abelian variety $A$ defined over $\mathbb{C}$ is not Zariski dense in a subvariety $V$ of $A$ unless $V$ is a translate of an algebraic subgroup of $A$ by a torsion point. Pink and others have suggested extending the Manin-Mumford conjecture to a more general question regarding unlikely intersections between a subvariety $V$ of a semiabelian scheme $A$ and algebraic subgroups of the fibers of $A$ having codimension greater than the dimension of $V$ [Bombieri et al. 1999; Habegger 2009; Masser and Zannier 2010; Masser and Zannier 2012; Pink 2005]. Here we state a special case of the question when $V$ is a curve:

Question 1.1. Let $\mathscr{Y}$ be a semiabelian scheme over a variety $9 y$ defined over $\mathbb{C}$, and let $V \subset \mathscr{Y}$ be a curve that is not contained in any proper algebraic subgroup of $\mathscr{Y}$. We define

$$
\varphi^{[2]}:=\bigcup_{y \in \mathcal{O}} B_{y},
$$

where $B_{y}$ is the union of all algebraic subgroups of the fiber $\mathscr{Y}_{y}$ of codimension at least equal to 2. Must the intersection of $V$ with $\mathscr{S}^{[2]}$ be finite?

Ghioca was partially supported by an NSERC Discovery Grant. Hsia was partially supported by the National Center of Theoretical Sciences of Taiwan and NSC grant 99-2115-M-008-007-MY3. Tucker was partially supported by NSF grants 0801072 and 0854839.

MSC2010: primary 37P05; secondary 37P10.

Keywords: preperiodic points, heights. 
Bertrand [2011] recently showed that the answer to Question 1.1 is sometimes "no". The question may, however, have a positive answer in many instances. For example, Masser and Zannier [2010; 2012] study Question 1.1 when $\mathscr{Y}$ is the square of the Legendre family of elliptic curves $E_{\lambda}$ (over the base $\mathbb{A}^{1} \backslash\{0,1\}$ ) given by the equation $y^{2}=x(x-1)(x-\lambda)$. They show that for any two independent points $P$ and $Q$ on the generic fiber, there are at most finitely many $\lambda \in \mathbb{C}$ such that the specializations $P_{\lambda}$ and $Q_{\lambda}$ are both torsion points for $E_{\lambda}$. Their work thus gives a positive answer to Question 1.1 in this special case.

The result of Masser and Zannier has a distinct dynamical flavor. Indeed, one may consider the following more general problem. Let $\left\{X_{\lambda}\right\}$ be an algebraic family of quasiprojective varieties defined over $\mathbb{C}$, let $\Phi_{\lambda}: X_{\lambda} \rightarrow X_{\lambda}$ be an algebraic family of endomorphisms, and let $P_{\lambda} \in X_{\lambda}$ and $Q_{\lambda} \in X_{\lambda}$ be two algebraic families of points. Under what conditions do there exist infinitely many $\lambda$ such that both $P_{\lambda}$ and $Q_{\lambda}$ are preperiodic for $\Phi_{\lambda}$ ? Indeed, the problem from [Masser and Zannier 2010; 2012] fits into this general dynamical framework by letting $X_{\lambda}=E_{\lambda}$ be the Legendre family of elliptic curves and letting $\Phi_{\lambda}$ be the multiplication-by-2 map on each elliptic curve in this family.

Baker and DeMarco [2011] study an interesting special case of the general dynamical question above first suggested by Zannier at an American Institute of Mathematics workshop in 2008. Given complex numbers $a$ and $b$ and an integer $d \geq 2$, when do there exist infinitely many $\lambda \in \mathbb{C}$ such that both $a$ and $b$ are preperiodic for the action of $f_{\lambda}(x):=x^{d}+\lambda$ on $\mathbb{C}$ ? They show that this happens if and only if $a^{d}=b^{d}$. We prove this generalization of the main result of [Baker and DeMarco 2011]:

Theorem 1.2. Let $f \in \mathbb{C}[x]$ be any polynomial of degree $d \geq 2$, and let $a, b \in \mathbb{C}$. Then there exist infinitely many $\lambda \in \mathbb{C}$ such that both $a$ and $b$ are preperiodic for $f(x)+\lambda$ if and only if $f(a)=f(b)$.

We will derive Theorem 1.2 from a more technical result, Theorem 2.3, which also treats the case of "nonconstant starting points" $\boldsymbol{a}$ and $\boldsymbol{b}$, a topic that was raised in [Baker and DeMarco 2011].

One might hope to formulate a general dynamical version of Question 1.1 for polarizable endomorphisms of projective varieties more general than multiplicationby- $m$ maps on abelian varieties (an endomorphism $\Phi$ of a projective variety $X$ is polarizable if there exists $d \geq 2$ and a line bundle $\mathscr{L}$ on $X$ such that $\Phi^{*}(\mathscr{L})$ is linearly equivalent to $\mathscr{L}^{\otimes d}$ in $\operatorname{Pic}(X)$ ) by using the analogy between abelian subschemes and preperiodic subvarieties. Because of the results of Baker and DeMarco, along with the results of this paper, we believe it is reasonable to ask the following dynamical analog of Question 1.1: 
Question 1.3. Let $Y$ be any quasiprojective curve defined over $\mathbb{C}$, and let $F$ be the function field of $Y$. Let $\boldsymbol{a}, \boldsymbol{b} \in \mathbb{P}^{1}(F)$, and let $V \subset \mathscr{X}:=\mathbb{P}_{F}^{1} \times_{F} \mathbb{P}_{F}^{1}$ be the $\mathbb{C}$-curve $(\boldsymbol{a}, \boldsymbol{b})$. Let $\boldsymbol{f}: \mathbb{P}^{1} \rightarrow \mathbb{P}^{1}$ be a rational map of degree $d \geq 2$ defined over $F$. Then for all but finitely many $\lambda \in Y, f$ induces a well-defined rational map $f_{\lambda}: \mathbb{P}^{1} \rightarrow \mathbb{P}^{1}$ defined over $\mathbb{C}$. If there exist infinitely many $\lambda \in Y$ such that both $\boldsymbol{a}(\lambda)$ and $\boldsymbol{b}(\lambda)$ are preperiodic points of $\mathbb{P}^{1}(\mathbb{C})$ under the action of $f_{\lambda}$, then must $V$ be contained in a proper preperiodic subvariety of $\mathscr{L}$ under the action of $\Phi:=(f, f) ?$

Theorem 1.2 is a special case of Question 1.3 for $f_{\lambda}(x)=f(x)+\lambda$ and constant starting points $\boldsymbol{a}(\lambda)=a$ and $\boldsymbol{b}(\lambda)=b$. Theorem 2.3 also allows us to prove some other special cases of Question 1.3 such as the following:

Theorem 1.4. Let $f \in \mathbb{C}[x]$ be any polynomial of degree $d \geq 2$, let $g \in \mathbb{C}[x]$ be any nonconstant polynomial, and let $c \in \mathbb{C}^{*}$. Then there exist at most finitely many $\lambda \in \mathbb{C}$ such that either

(1) both $g(\lambda)$ and $g(\lambda+c)$ are preperiodic for $f(x)+\lambda$ or

(2) both $g(\lambda)$ and $g(\lambda)+c$ are preperiodic for $f(x)+\lambda$.

The next result is for the case when the family of maps $f$ is constant:

Theorem 1.5. Let $f \in \mathbb{C}[x]$ be a polynomial of degree $d \geq 2$, and let $\boldsymbol{a}, \boldsymbol{b} \in \mathbb{C}[\lambda]$ be two polynomials of same degree and with the same leading coefficient. If there exist infinitely many $\lambda \in \mathbb{C}$ such that both $\boldsymbol{a}(\lambda)$ and $\boldsymbol{b}(\lambda)$ are preperiodic for $f$, then $\boldsymbol{a}=\boldsymbol{b}$.

A special case of Theorem 1.5 is that for any fixed $c \in \mathbb{C}^{*}$, there can be only finitely many $\lambda \in \mathbb{C}$ such that both $\lambda$ and $\lambda+c$ are preperiodic for $f$. In fact, more generally it provides a positive answer to a special case of Zhang's dynamical Manin-Mumford conjecture, which states that for a polarizable endomorphism $\Phi: X \rightarrow X$ on a projective variety, the only subvarieties of $X$ containing a dense set of preperiodic points are those subvarieties that are themselves preperiodic under $f$; see [Zhang 1995, Conjecture 2.5; 2006, Conjecture 1.2.1, Conjecture 4.1.7] for details. This conjecture turns out to be false in general [Ghioca et al. 2011], but it may be true in many cases. For example, let $X:=\mathbb{P}^{1} \times \mathbb{P}^{1}, \Phi(x, y):=(f(x), f(y))$ for a polynomial $f$ of degree $d \geq 2$, and $Y$ be the Zariski closure in $X$ of the set $\{(\boldsymbol{a}(z), \boldsymbol{b}(z)): z \in \mathbb{C}\}$, where $\boldsymbol{a}, \boldsymbol{b} \in \mathbb{C}[x]$ are polynomials of same degree and with the same leading coefficient; Theorem 1.5 implies that if $Y$ contains infinitely many points preperiodic under $\Phi$, then $Y$ is the diagonal subvariety of $X$ and thus is itself preperiodic under $\Phi$. Theorem 1.5 also has consequences for a case of a revised dynamical Manin-Mumford conjecture [Ghioca et al. 2011, Conjecture 1.4]; see Section 11 for details.

The plan of our paper is as follows. In Section 2, we state our main result, Theorem 2.3, and some of its consequences and then describe the method of 
our proof. In Section 3, we set up our notation while in Section 4 we give a brief overview of Berkovich spaces. Then in Section 5, we introduce some basic preliminaries regarding the iterates of a generic starting point $c$ under a family of maps $f$. Section 6 contains computations of the capacities of the generalized $v$-adic Mandelbrot sets associated with a generic point $c$ under the action of $\boldsymbol{f}$. In Section 7, we prove an explicit formula for the Green function for the generalized $v$-adic Mandelbrot sets when $v$ is an archimedean valuation. We proceed with our proof of the direct implication in Theorem 2.3 in Section 8 (for the case $f_{\lambda} \in \overline{\mathbb{Q}}[x]$ and $\boldsymbol{a}, \boldsymbol{b} \in \overline{\mathbb{Q}}[x]$ ) and in Section 10 (for the general case). In Section 9, we prove the converse implication from Theorem 2.3. Then in Section 11, we conclude our paper by proving Corollary 2.7 and discussing the connections between our Question 1.3 and the dynamical Manin-Mumford conjecture formulated by Ghioca, Tucker, and Zhang [2011].

\section{Statement of the main results}

A special case of Question 1.3 is when $Y=\mathbb{A}^{1}, f \in R[x]$, where $R=\mathbb{C}[\lambda]$, and $\boldsymbol{a}, \boldsymbol{b} \in R$. In Theorem 2.3, we provide a positive answer to Question 1.3 for any family of polynomials of the form

$$
f_{\lambda}(x)=x^{d}+\sum_{i=0}^{d-2} c_{i}(\lambda) x^{i}, \quad \text { where } c_{i}(\lambda) \in \mathbb{C}[\lambda] \text { for } i=0, \ldots, d-2,
$$

together with some mild restriction on the polynomials $\boldsymbol{a}$ and $\boldsymbol{b}$.

We say that a polynomial $f(x)$ of degree $d$ is in normal form if it is monic and its coefficient of $x^{d-1}$ equals 0 . (Note that any polynomial of degree $d>1$ can be put in normal form after a change of coordinates.) As a matter of notation, we rewrite (2.1) as

$$
f_{\lambda}(x)=P(x)+\sum_{i=1}^{r} Q_{i}(x) \cdot \lambda^{m_{i}}
$$

for some polynomial $P \in \mathbb{C}[x]$ in normal form of degree $d$, some nonnegative integer $r$, integers $m_{0}:=0<m_{1}<\cdots<m_{r}$, and some polynomials $Q_{i} \in \mathbb{C}[x]$ of degrees $0 \leq e_{i} \leq d-2$. We do not exclude the case $r=0$, in which case the sum in the sigma notation is empty and $\left\{f_{\lambda}\right\}_{\lambda}$ is a constant family of polynomials.

Let $\boldsymbol{a}(\lambda), \boldsymbol{b}(\lambda) \in \mathbb{C}[\lambda]$. If $\boldsymbol{a}$ is preperiodic for $\boldsymbol{f}$, that is, $\boldsymbol{f}^{k}(\boldsymbol{a})=\boldsymbol{f}^{\ell}(\boldsymbol{a})$ for some $k \neq \ell$, then for each $\boldsymbol{b}$, one can show that there are infinitely many $\lambda \in \mathbb{C}$ such that $\boldsymbol{b}(\lambda)$ (and thus also $\boldsymbol{a}(\lambda)$ ) is preperiodic for $f_{\lambda}$ (see also Proposition 9.1). Therefore, we may assume that $\boldsymbol{a}$ and $\boldsymbol{b}$ are not preperiodic for $\boldsymbol{f}$. Assuming there exist infinitely many $\lambda \in \mathbb{C}$ such that both $\boldsymbol{a}(\lambda)$ and $\boldsymbol{b}(\lambda)$ are preperiodic for $f_{\lambda}$, then Question 1.3 predicts that there exist $\varphi_{1}$ and $\varphi_{2}$ commuting with $\boldsymbol{f}$ such that 
$\varphi_{1}(\boldsymbol{a})=\varphi_{2}(\boldsymbol{b})$. A natural possibility is for $\varphi_{1}$ and $\varphi_{2}$ to be iterates of $\boldsymbol{f}$; under a mild condition on $\boldsymbol{a}$ and $\boldsymbol{b}$, we prove that this is the only possibility.

Theorem 2.3. Let $\boldsymbol{f}:=f_{\lambda}$ be the family of one-parameter polynomials (indexed by all $\lambda \in \mathbb{C}$ ) given by

$$
f_{\lambda}(x):=x^{d}+\sum_{i=0}^{d-2} c_{i}(\lambda) x^{i}=P(x)+\sum_{j=1}^{r} Q_{j}(x) \cdot \lambda^{m_{j}}
$$

as above (see (2.1) and (2.2)). Let $\boldsymbol{a}, \boldsymbol{b} \in \mathbb{C}[\lambda]$, and assume there exist nonnegative integers $k$ and $\ell$ such that the following conditions hold:

(i) $f_{\lambda}^{k}(\boldsymbol{a}(\lambda))$ and $f_{\lambda}^{\ell}(\boldsymbol{b}(\lambda))$ have the same degree and the same leading coefficient as polynomials in $\lambda$, and

(ii) if $m=\operatorname{deg}_{\lambda}\left(f_{\lambda}^{k}(\boldsymbol{a}(\lambda))\right) \operatorname{deg}_{\lambda}\left(f_{\lambda}^{\ell}(\boldsymbol{b}(\lambda))\right)$, then $m \geq m_{r}$.

Then there exist infinitely many $\lambda \in \mathbb{C}$ such that both $\boldsymbol{a}(\lambda)$ and $\boldsymbol{b}(\lambda)$ are preperiodic points for $f_{\lambda}$ if and only if $f_{\lambda}^{k}(\boldsymbol{a}(\lambda))=f_{\lambda}^{\ell}(\boldsymbol{b}(\lambda))$.

Remarks 2.4. (a) The one-dimensional $\mathbb{C}$-scheme $(\boldsymbol{a}, \boldsymbol{b}) \subset \mathscr{X}:=\mathbb{P}_{\mathbb{C}(\lambda)}^{1} \times \mathbb{C}(\lambda) \mathbb{P}_{\mathbb{C}(\lambda)}^{1}$ in Theorem 2.3 is contained in the two-dimensional $\mathbb{C}$-subscheme $\mathscr{Y}$ of $\mathscr{X}$ given by the equation

$$
\boldsymbol{f}^{k}(x)=\boldsymbol{f}^{\ell}(y)
$$

where $(x, y)$ are the coordinates of $\mathscr{X}$. Such a $\mathscr{y}$ is fixed by the action of $(\boldsymbol{f}, \boldsymbol{f})$ on $\mathscr{X}$ as predicted by Question 1.3.

(b) It follows from the Lefschetz principle that the same statements in Theorem 2.3 hold if we replace $\mathbb{C}$ by any other algebraically closed complete valued field of characteristic 0 .

(c) We note that if $c \in \mathbb{C}[\lambda]$ has the property that there exists $k \in \mathbb{N}$ such that $\operatorname{deg}_{\lambda}\left(f_{\lambda}^{k}(\boldsymbol{c}(\lambda))\right)=m$ has the property (ii) from Theorem 2.3, then $\boldsymbol{c}$ is not preperiodic for $f$ (see Lemma 5.2).

(d) If $\boldsymbol{f}$ is not a constant family, then it follows from Benedetto's theorem [2005] that $c \in \mathbb{C}[\lambda]$ is not preperiodic for $\boldsymbol{f}$ if and only if there exists $k \in \mathbb{N}$ such that $\operatorname{deg}_{\lambda}\left(f_{\lambda}^{k}(\boldsymbol{c}(\lambda))\right) \geq m_{r}$. On the other hand, if $\boldsymbol{f}$ is a constant family of polynomials defined over $\mathbb{C}$, that is, $r=0$ and $m_{0}=0$ in Theorem 2.3 , then implicitly $m>0$. (Otherwise the conclusion holds trivially.)

Theorem 2.3 generalizes known results regarding "unlikely intersections" in the dynamical setting including the dynamical Manin-Mumford questions (see Section 11). First, Theorem 2.3 generalizes the main result of [Baker and DeMarco 2011] in two ways. On one hand, in the case when $\boldsymbol{a}$ and $\boldsymbol{b}$ are both constant, we can prove a generalization of the main result from [ibid.] as follows: 
Theorem 2.5. Let $a, b \in \mathbb{C}$, let $d \geq 2$, and let $c_{0}, \ldots, c_{d-2} \in \mathbb{C}[\lambda]$ such that $\operatorname{deg}\left(c_{0}\right)>\operatorname{deg}\left(c_{i}\right)$ for each $i=1, \ldots, d-2$. If there are infinitely many $\lambda \in \mathbb{C}$ such that both $a$ and $b$ are preperiodic for

$$
f_{\lambda}(x):=x^{d}+\sum_{i=0}^{d-2} c_{i}(\lambda) x^{i},
$$

then $f_{\lambda}(a)=f_{\lambda}(b)$.

Proof. We apply Theorem 2.3 for $\boldsymbol{a}(\lambda):=f_{\lambda}(a)$ and $\boldsymbol{b}(\lambda):=f_{\lambda}(b)$.

Consequently, Theorem 2.5 yields the proof of Theorem 1.2.

Proof of Theorem 1.2. Note that in this case, we may drop the hypothesis that $f(x)$ is in normal form since we may conjugate $f(x)$ by some linear polynomial $\delta \in \mathbb{C}[x]$ such that $g:=\delta^{-1} \circ f \circ \delta+\delta^{-1}(\lambda)$ is a family of polynomials in normal form. Then apply Theorem 2.5 to the pair of points $\delta^{-1}(a)$ and $\delta^{-1}(b)$.

On the other hand, using our Theorem 2.3 we are able to treat the case when the pair of points $\boldsymbol{a}$ and $\boldsymbol{b}$ depend algebraically on the parameter. This answers a question raised by Silverman mentioned in [Baker and DeMarco 2011, Section 1.1]. For instance, as an application of Theorem 2.3, by taking $f=f(x)+\lambda$ for any nonconstant polynomial $f(x) \in \mathbb{C}[x]$ of degree at least 2 , we have the following:

Corollary 2.6. Let $f \in \mathbb{C}[x]$ be any polynomial of degree $d \geq 2$, and let $\boldsymbol{a}, \boldsymbol{b} \in \mathbb{C}[\lambda]$ be polynomials such that $\boldsymbol{a}$ and $\boldsymbol{b}$ have the same degree and the same leading coefficient. Then there are infinitely many $\lambda \in \mathbb{C}$ such that both $\boldsymbol{a}(\lambda)$ and $\boldsymbol{b}(\lambda)$ are preperiodic under the action of $f(x)+\lambda$ if and only if $\boldsymbol{a}(\lambda)=\boldsymbol{b}(\lambda)$.

Proof. First, the theorem is vacuously true if $\boldsymbol{a}$ and $\boldsymbol{b}$ are constant polynomials since then they are automatically equal because they have the same leading coefficient. So we may assume that $\operatorname{deg}(\boldsymbol{a})=\operatorname{deg}(\boldsymbol{b}) \geq 1$.

Second, we conjugate $f(x)$ by some linear polynomial $\delta \in \mathbb{C}[x]$ such that $g:=\delta^{-1} \circ f \circ \delta$ is a polynomial in normal form. Then we apply Theorem 2.3 to the family of polynomials $g(x)+\delta^{-1}(\lambda)$ and to the starting points $\delta^{-1}(\boldsymbol{a}(\lambda))$ and $\delta^{-1}(\boldsymbol{b}(\lambda))$. Since $\boldsymbol{a}$ and $\boldsymbol{b}$ are polynomials of the same positive degree and same leading coefficient, it is immediate to check that conditions (i) and (ii) of Theorem 2.3 hold for $k=\ell=0$. Therefore, $\boldsymbol{a}(\lambda)=\boldsymbol{b}(\lambda)$ as desired.

An important special case of Corollary 2.6 is Theorem 1.4. Using Theorem 2.3 when $\boldsymbol{f}$ is a constant family of polynomials, we obtain a proof of Theorem 1.5.

Proof of Theorem 1.5. The result is an immediate consequence of Theorem 2.3 once we observe, as before, that we may replace $f$ with a conjugate $\delta^{-1} \circ f \circ \delta$ of itself that is a polynomial in normal form. (Note that in this case, we also replace $\boldsymbol{a}$ and $\boldsymbol{b}$ by $\delta^{-1}(\boldsymbol{a})$ and $\delta^{-1}(\boldsymbol{b})$, respectively, which are also polynomials in $\lambda$ of the same degree and same leading coefficient.) 
On the other hand, assuming each $c_{i}$ and also $\boldsymbol{a}$ and $\boldsymbol{b}$ have algebraic coefficients, the exact same proof we have yields stronger statements of Theorems 2.3, 1.5, and Corollary 2.6, allowing us to replace the hypothesis that there are infinitely many $\lambda \in \overline{\mathbb{Q}}$ such that both $\boldsymbol{a}(\lambda)$ and $\boldsymbol{b}(\lambda)$ are preperiodic for $f_{\lambda}$ with the weaker condition that there exists an infinite sequence of $\lambda_{n} \in \overline{\mathbb{Q}}$ such that

$$
\lim _{n \rightarrow \infty} \hat{h}_{f_{\lambda_{n}}}\left(\boldsymbol{a}\left(\lambda_{n}\right)\right)+\hat{h}_{f_{\lambda_{n}}}\left(\boldsymbol{b}\left(\lambda_{n}\right)\right)=0
$$

where for each $\lambda \in \overline{\mathbb{Q}}, \hat{h}_{f_{\lambda}}$ is the canonical height constructed with respect to the polynomial $f_{\lambda}$. (For the precise definition of the canonical height with respect to a polynomial map, see Section 3.) Therefore, we can prove a special case of Zhang's dynamical Bogomolov conjecture [2006].

Corollary 2.7. Let $Y \subset \mathbb{P}^{1} \times \mathbb{P}^{1}$ be a curve that admits a parametrization given by $(\boldsymbol{a}(z), \boldsymbol{b}(z))$ for $z \in \mathbb{C}$, where $\boldsymbol{a}, \boldsymbol{b} \in \overline{\mathbb{Q}}[x]$ are polynomials of the same degree and with the same leading coefficient. Let $f \in \overline{\mathbb{Q}}[x]$ be a polynomial of degree at least equal to 2 , and let $\Phi(x, y):=(f(x), f(y))$ be the diagonal action of $f$ on $\mathbb{P}^{1} \times \mathbb{P}^{1}$. If there exists an infinite sequence of points $\left(x_{n}, y_{n}\right) \in Y(\overline{\mathbb{Q}})$ such that

$$
\lim _{n \rightarrow \infty} \hat{h}_{f}\left(x_{n}\right)+\hat{h}_{f}\left(y_{n}\right)=0,
$$

then $\boldsymbol{a}=\boldsymbol{b}$. In particular, $Y$ is the diagonal subvariety of $\mathbb{P}^{1} \times \mathbb{P}^{1}$ and thus is preperiodic under the action of $\Phi$.

Remark 2.8. In fact, this result holds not only over $\overline{\mathbb{Q}}$ but also over the algebraic closure of any global function field $L$ (whose subfield of constants is $K$ ) as long as $f$ is not conjugate to a polynomial with coefficients in $\bar{K}$.

Note that the second author, together with Baker, proved a similar result [Baker and Hsia 2005, Theorem 8.10] in the case when $Y$ is a line; that is, if a line in $\mathbb{P}^{1} \times \mathbb{P}^{1}$ contains an infinite set of points of small canonical height with respect to the coordinatewise action of the polynomial $f$ on $\mathbb{P}^{1} \times \mathbb{P}^{1}$, then the line $Y$ is preperiodic under the action of $(f, f)$ on $\mathbb{P}^{1} \times \mathbb{P}^{1}$.

Laura DeMarco communicated to us that our Theorem 2.3 yields the proof of the first case of a conjecture she made as a dynamical analogue of the AndréOort conjecture. Essentially, the dynamical André-Oort conjecture envisioned by DeMarco aims to characterize subvarieties in the moduli space $M_{d}$ of complex rational maps $f: \mathbb{P}^{1} \rightarrow \mathbb{P}^{1}$ (of degree $d>1$ ) that contain a Zariski dense subset of postcritically finite rational maps. A rational map is postcritically finite (PCF) if all of its critical points are preperiodic. The PCF rational maps play an important role in complex dynamics; for example, the Lattès maps are PCF. 
Our Theorem 2.3 has the following consequence. Let $f=f_{\lambda}$ be a family of polynomials in normal form of degree $d$ with polynomial coefficients in $\lambda$. Furthermore, assume the critical points $\boldsymbol{c}_{1}(\lambda), \ldots, \boldsymbol{c}_{d-1}(\lambda)$ of $f_{\lambda}$ are also polynomials in $\lambda$. Let $I$ be the collection of indices $i$ such that $c_{i}$ is not preperiodic for $f$. Suppose for each $i \in I$, there exist iterates $f_{\lambda}^{m_{i}}\left(\boldsymbol{c}_{i}(\lambda)\right)$ with the same degree and leading coefficients in $\lambda$ and that this degree is large enough (that is, satisfies the hypothesis from Theorem 2.3). Then there are infinitely many PCF maps in this family if and only if all $\boldsymbol{f}^{m_{i}}\left(\boldsymbol{c}_{i}\right)$ (for $i \in I$ ) are equal.

We prove Theorem 2.3 first for the case when both $\boldsymbol{a}$ and $\boldsymbol{b}$ and also each of the $c_{i}$ have algebraic coefficients, and then we extend our proof to the general case. For the extension to $\mathbb{C}$, we use a result of Benedetto [2005] (see also Baker's extension [2009] to arbitrary rational maps), which states that for a polynomial $f$ of degree at least equal to 2 defined over a function field $K$ of finite transcendence degree over a subfield $K_{0}$, if $f$ is not isotrivial (that is, $f$ is not conjugate to a polynomial defined over $\overline{K_{0}}$ ), then each $x \in \bar{K}$ is preperiodic if and only if its canonical height $\hat{h}_{f}(x)$ equals 0 . Strictly speaking, Benedetto's result is stated for function fields of transcendence degree 1, but a simple inductive argument on the transcendence degree yields the result for function fields of arbitrary finite transcendence degree. (See also [Baker 2009, Corollary 1.8], where Baker extends Benedetto's result to rational maps defined over function fields of arbitrary finite transcendence degree.)

Our results and proofs are inspired by the results of [Baker and DeMarco 2011] so that the strategy for the proof of Theorem 2.3 essentially follows their ideas. However, there are significantly more technical difficulties in our proofs. The plan of our proof is to use the $v$-adic generalized Mandelbrot sets introduced therein for the family of polynomials $f_{\lambda}$ and then use the equidistribution result of Baker and Rumely [2010]. A key ingredient is Proposition 6.8, which says that the canonical local height of the point in question at the place $v$ is a constant multiple of the Green function associated with the $v$-adic generalized Mandelbrot set. Then the condition that $\boldsymbol{a}(\lambda)$ and $\boldsymbol{b}(\lambda)$ are preperiodic is translated to the condition that the heights $h_{\mathbb{M}_{a}}(\lambda)$ and $h_{\mathbb{M}_{b}}(\lambda)$, respectively, are zero for the corresponding parameter $\lambda$. Therefore, the equidistribution result of Baker-Rumely can be applied to conclude that the $v$-adic generalized Mandelbrot sets for $\boldsymbol{a}(\lambda)$ and $\boldsymbol{b}(\lambda)$ are the same for each place $v$. Finally, we need to use an explicit formula for the Green function associated with the $v$-adic generalized Mandelbrot set corresponding to an archimedean valuation $v$ to conclude that the desired equality of $f_{\lambda}^{k}(\boldsymbol{a}(\lambda))$ and $f_{\lambda}^{\ell}(\boldsymbol{b}(\lambda))$ holds. Extra work is needed for the explicit description of the Green function for a $v$-adic generalized Mandelbrot set (when $v$ is an archimedean place) due to the fact that in our case, the polynomial $f_{\lambda}$ has arbitrary (finitely) many critical points that vary with $\lambda$ in contrast to the family of polynomials $x^{d}+\lambda$ from [Baker and DeMarco 2011], which has only one critical point for the entire family. 


\section{Notation and preliminary}

For any quasiprojective variety $X$ endowed with an endomorphism $\Phi$, we call a point $x \in X$ preperiodic if there exist two distinct nonnegative integers $m$ and $n$ such that $\Phi^{m}(x)=\Phi^{n}(x)$, where by $\Phi^{i}$ we always denote the $i$-iterate of the endomorphism $\Phi$. If $n=0$, then, by convention, $\Phi^{0}$ is the identity map.

Let $K$ be a field of characteristic 0 equipped with a set of inequivalent absolute values (places) $\Omega_{K}$, normalized so that the product formula holds; more precisely, for each $v \in \Omega_{K}$ there exists a positive integer $N_{v}$ such that for all $\alpha \in K^{*}$ we have $\prod_{v \in \Omega}|\alpha|_{v}^{N_{v}}=1$, where for $v \in \Omega_{K}$, the corresponding absolute value is denoted by $|\cdot|_{v}$. Let $\mathbb{C}_{v}$ be a fixed completion of the algebraic closure of a completion of $\left(K,|\cdot|_{v}\right)$. When $v$ is an archimedean valuation, then $\mathbb{C}_{v}=\mathbb{C}$. We fix an extension of $|\cdot|_{v}$ to an absolute value of $\left(\mathbb{C}_{v},|\cdot|_{v}\right)$. Examples of product formula fields (or global fields) are number fields and function fields of projective varieties that are regular in codimension 1 [Lang 1983, Section 2.3; Bombieri and Gubler 2006, Section 1.4.6].

Let $f \in \mathbb{C}_{v}[x]$ be any polynomial of degree $d \geq 2$. Following Call and Silverman [1993], for each $x \in \mathbb{C}_{v}$, we define the local canonical height of $x$ by

$$
\hat{h}_{f, v}(x):=\lim _{n \rightarrow \infty} \frac{\log ^{+}\left|f^{n}(x)\right|_{v}}{d^{n}},
$$

where $\log ^{+} z$ always denotes $\log \max \{z, 1\}$ (for any real number $z$ ).

It is immediate that $\hat{h}_{f, v}\left(f^{i}(x)\right)=d^{i} \hat{h}_{f, v}(x)$, and thus, $\hat{h}_{f, v}(x)=0$ whenever $x$ is a preperiodic point for $f$. If $v$ is nonarchimedean and $f(x)=\sum_{i=0}^{d} a_{i} x^{i}$, then $|f(x)|_{v}=\left|a_{d} x^{d}\right|_{v}>|x|_{v}$ when $|x|_{v}>r_{v}$, where

$$
r_{v}:=\max \left\{\left|a_{d}\right|_{v}^{-1 /(d-1)} \max _{0 \leq i<d}\left\{\left|\frac{a_{i}}{a_{d}}\right|^{1 /(d-i)}\right\}\right\} .
$$

Moreover, if $|x|_{v}>r_{v}$, then $\hat{h}_{v}(x)=\log |x|_{v}+\log \left|a_{d}\right|_{v} /(d-1)>0$. For more details, see [Ghioca and Tucker 2008; Hsia 2008]. (Although these results are for canonical heights associated with Drinfeld modules, all the proofs go through for any local canonical height associated with any polynomial with respect to any nonarchimedean place.)

Now, if $v$ is archimedean, again it is easy to see that if $|x|_{v}$ is sufficiently large, then $|f(x)|_{v} \gg|x|_{v}^{d}$, and moreover, $\left|f^{n}(x)\right|_{v} \rightarrow \infty$ as $n \rightarrow \infty$.

We fix an algebraic closure $\bar{K}$ of $K$, and for each $v \in \Omega_{K}$ we fix an embedding $\bar{K} \hookrightarrow \mathbb{C}_{v}$. Assume $f \in \bar{K}[x]$. Call and Silverman [1993] also defined the global canonical height $\hat{h}(x)$ for each $x \in \bar{K}$ as

$$
\hat{h}_{f}(x)=\lim _{n \rightarrow \infty} \frac{h\left(f^{n}(x)\right)}{d^{n}},
$$


where $h$ is the usual (logarithmic) Weil height on $\bar{K}$. Call and Silverman show that the global canonical height decomposes into a sum of the corresponding local canonical heights.

For each $\sigma \in \operatorname{Gal}(\bar{K} / K)$, we denote by $\hat{h}_{f^{\sigma}}$ the global canonical height computed with respect to $f^{\sigma}$, which is the polynomial obtained by applying $\sigma$ to each coefficient of $f$. Similarly, for each $v \in \Omega_{K}$ we denote by $\hat{h}_{f^{\sigma}, v}$ the corresponding local canonical height constructed with respect to the polynomial $f^{\sigma}$. For $x \in \bar{K}$, we have $\hat{h}_{f}(x)=0$ if and only if $\hat{h}_{f^{\sigma}}\left(x^{\sigma}\right)=0$ for all $\sigma \in \operatorname{Gal}(\bar{K} / K)$. More precisely, for $x \in \bar{K}$ we have

$$
\hat{h}_{f}(x)=0 \Longleftrightarrow \hat{h}_{f^{\sigma}, v}\left(x^{\sigma}\right)=0 \text { for all } v \in \Omega_{K} \text { and all } \sigma \in \operatorname{Gal}(\bar{K} / K) .
$$

Essentially, (3.2) says that $\hat{h}_{f}(x)=0$ if and only if the orbits of $x^{\sigma}$ under each polynomial $f^{\sigma}$ (for $\sigma \in \operatorname{Gal}(\bar{K} / K)$ ) are bounded with respect to each absolute value $|\cdot|_{v}$ for $v \in \Omega_{K}$.

Benedetto [2005] proved that if a polynomial $f$ defined over a function field $K$ (endowed with a set $\Omega_{K}$ of absolute values) is not isotrivial (that is, it cannot be conjugated to a polynomial defined over the constant subfield of $K$ ), then each point $c \in \bar{K}$ is preperiodic for $f$ if and only if its global canonical height (computed with respect to $f$ ) equals 0 . In particular, if $c \in \bar{K}$, then $c$ is preperiodic if and only if

$$
\hat{h}_{f^{\sigma}, v}\left(c^{\sigma}\right)=0 \quad \text { for all } \sigma \in \operatorname{Gal}(\bar{K} / K) \text { and for all places } v \in \Omega_{K} .
$$

Let

$$
\boldsymbol{f}=f_{\lambda}:=x^{d}+\sum_{i=0}^{d-2} c_{i}(\lambda) x^{i},
$$

where $c_{i}(\lambda) \in \mathbb{C}[\lambda]$ for $i=0, \ldots, d-2$, and let $c(\lambda) \in \mathbb{C}[\lambda]$. We let $K$ be the field extension of $\mathbb{Q}$ generated by all coefficients of each $c_{i}(\lambda)$ and of $\boldsymbol{c}(\lambda)$. Assume $K$ is a global field; that is, it has a set $\Omega_{K}$ of inequivalent absolute values with respect to which the nonzero elements of $K$ satisfy a product formula. For each place $v \in \Omega_{K}$, we define the $v$-adic Mandelbrot set $M_{\boldsymbol{c}, v}$ for $\boldsymbol{c}$ with respect to the family of polynomials $\boldsymbol{f}$ as the set of all $\lambda \in \mathbb{C}_{v}$ such that $\hat{h}_{f_{\lambda}, v}(\boldsymbol{c}(\lambda))=0$, that is, the set of all $\lambda \in \mathbb{C}_{v}$ such that the iterates $f_{\lambda}^{n}(\boldsymbol{c}(\lambda))$ are bounded with respect to the $v$-adic absolute value.

\section{Berkovich spaces}

We now introduce Berkovich spaces and state the equidistribution theorem of Baker and Rumely [2010], which will be key for the proofs of Theorems 2.3 and 2.5.

Let $K$ be a global field of characteristic 0 , and let $\Omega_{K}$ be the set of its inequivalent absolute values. For each $v \in \Omega_{K}$, we let $\mathbb{C}_{v}$ be the completion of an algebraic closure of the completion of $K$ at $v$. Let $\mathbb{A}_{\text {Berk, } \mathbb{C}_{v}}^{1}$ denote the Berkovich affine 
line over $\mathbb{C}_{v}$; see [Berkovich 1990; Baker and Rumely 2010, Section 2.1] for details. Then $\mathbb{A}_{\mathrm{Berk}, \mathbb{C}_{v}}^{1}$ is a locally compact, Hausdorff, path-connected space containing $\mathbb{C}_{v}$ as a dense subspace (with the topology induced from the $v$-adic absolute value). As a topological space, $\mathbb{A}_{\mathrm{Berk}, \mathbb{C}_{v}}^{1}$ is the set consisting of all multiplicative seminorms, denoted by $[\cdot]_{x}$, on $\mathbb{C}_{v}[T]$ extending the absolute value $|\cdot|_{v}$ on $\mathbb{C}_{v}$ endowed with the weakest topology such that the map $z \mapsto[f]_{z}$ is continuous for all $f \in \mathbb{C}_{v}[T]$. It follows from the Gelfand-Mazur theorem that if $\mathbb{C}_{v}$ is the field of complex numbers $\mathbb{C}$, then $\mathbb{A}_{\text {Berk, } \mathbb{C}}^{1}$ is homeomorphic to $\mathbb{C}$. In the following, we will also use $\mathbb{A}_{\text {Berk, } \mathbb{C}_{v}}^{1}$ to denote the complex line $\mathbb{C}$ whenever $\mathbb{C}_{v}=\mathbb{C}$. If $\left(\mathbb{C}_{v},|\cdot|_{v}\right)$ is nonarchimedean, then the set of seminorms can be described as follows. If $\left\{D\left(a_{i}, r_{i}\right)\right\}_{i}$ is any decreasing nested sequence of closed disks $D\left(c_{i}, r_{i}\right)$ centered at points $c_{i} \in \mathbb{C}_{v}$ of radius $r_{i} \geq 0$, then the map $f \mapsto \lim _{i \rightarrow \infty}[f]_{D\left(c_{i}, r_{i}\right)}$ defines a multiplicative seminorm on $\mathbb{C}_{v}[T]$, where $[f]_{D\left(c_{i}, r_{i}\right)}$ is the sup norm of $f$ over the closed disk $D\left(a_{i}, r_{i}\right)$. Berkovich's classification theorem says that there are exactly four types of points: types I, II, III, and IV. The first three can be described in terms of closed disks $\zeta:=D(c, r)=\bigcap D\left(c_{i}, r_{i}\right)$, where $c \in \mathbb{C}_{v}$ and $r \geq 0$. The corresponding multiplicative seminorm is just $f \mapsto[f]_{D(c, r)}$ for $f \in \mathbb{C}_{v}[T]$. Then $\zeta$ is of type $\mathrm{I}$, II or III if and only if $r=0, r \in\left|\mathbb{C}_{v}^{*}\right|_{v}$ or $r \notin\left|\mathbb{C}_{v}^{*}\right|_{v}$, respectively. As for type IV points, they correspond to sequences of decreasing nested disks $D\left(c_{i}, r_{i}\right)$ such that $\bigcap D\left(c_{i}, r_{i}\right)=\varnothing$ and the multiplicative seminorm is $f \mapsto \lim _{i \rightarrow \infty}[f]_{D\left(c_{i}, r_{i}\right)}$ as described above. For details, see [Berkovich 1990; Baker and Rumely 2010]. For $\zeta \in \mathbb{A}_{\text {Berk, } \mathbb{C}_{v}}^{1}$, we sometimes write $|\zeta|_{v}$ instead of $[T]_{\zeta}$.

In order to apply the main equidistribution result from [Baker and Rumely 2010, Theorem 7.52], we recall the potential theory on the affine line over $\mathbb{C}_{v}$. We will focus on the case when $\mathbb{C}_{v}$ is a nonarchimedean field; the case $\mathbb{C}_{v}=\mathbb{C}$ is classical. (We refer the reader to [Ransford 1995].) The right setting for nonarchimedean potential theory is the potential theory on $\mathbb{A}_{\text {Berk, } \mathbb{C}_{v}}^{1}$ developed in [Baker and Rumely 2010]. We quote part of a nice summary of the theory from [Baker and DeMarco 2011, Section 2.2 and Section 2.3] without going into details. We refer the reader to [Baker and DeMarco 2011; Baker and Rumely 2010] for all the details and proofs. Let $E$ be a compact subset of $\mathbb{A}_{\text {Berk, } \mathbb{C}_{v}}^{1}$. Then analogous to the complex case, the Green function $G_{E}$ of $E$ relative to $\infty$ and the logarithmic capacity $\gamma(E):=e^{-V(E)}$ can be defined, where $V(E)$ is the infimum of the energy integral with respect to all possible probability measures supported on $E$. More precisely,

$$
V(E)=\inf _{\mu} \iint_{E \times E}-\log \delta(x, y) d \mu(x) d \mu(y),
$$

where the infimum is computed with respect to all probability measures $\mu$ supported on $E$ while for $x, y \in \mathbb{A}_{\text {Berk, } \mathbb{C}_{v}}^{1}$, the function $\delta(x, y)$ is the Hsia kernel [Baker and 
Rumely 2010, Proposition 4.1]:

$$
\delta(x, y):=\limsup _{\substack{z, w \in \mathbb{C}_{v} \\ z \rightarrow x, w \rightarrow y}}|z-w|_{v} .
$$

The following are basic properties of the logarithmic capacity of $E$.

- If $E_{1}$ and $E_{2}$ are two compact subsets of $\mathbb{A}_{\text {Berk, } \mathbb{C}_{v}}^{1}$ such that $E_{1} \subset E_{2}$, then $\gamma\left(E_{1}\right) \leq \gamma\left(E_{2}\right)$.

- If $E=\{\zeta\}$, where $\zeta$ is a type II or III point corresponding to a closed disk $D(c, r)$, then $\gamma(E)=r>0$ [Baker and Rumely 2010, Example 6.3]. (This can be viewed as an analogue of the fact that a closed disk $D(c, r)$ of positive radius $r$ in $\mathbb{C}_{v}$ has logarithmic capacity $\gamma(D(c, r))=r$.)

If $\gamma(E)>0$, then there exists a unique probability measure $\mu_{E}$ attaining the infimum of the energy integral. Furthermore, the support of $\mu_{E}$ is contained in the boundary

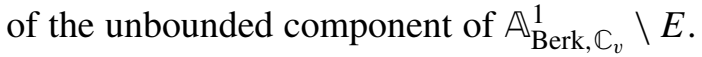

The Green function $G_{E}(z)$ of $E$ relative to infinity is a well-defined nonnegative real-valued subharmonic function on $\mathbb{A}_{\text {Berk, } \mathbb{C}_{v}}^{1}$ that is harmonic on $\mathbb{A}_{\text {Berk, } \mathbb{C}_{v}}^{1} \backslash E$ (in the sense of [Baker and Rumely 2010, Chapter 8] for the nonarchimedean setting; see [Ransford 1995] for the archimedean case). If $\gamma(E)=0$, then there exists no Green function associated with the set $E$ (see [Ransford 1995, Exercise 1, page 115] in the case when $|\cdot|_{v}$ is archimedean; a similar argument works when $|\cdot|_{v}$ is nonarchimedean). Indeed, as shown in [Baker and Rumely 2010, Proposition 7.17, page 151], if $\gamma(\partial E)=0$, then there exists no nonconstant harmonic function on

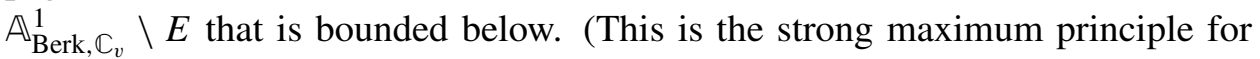
harmonic functions defined on Berkovich spaces). The following result is [Baker and DeMarco 2011, Lemmas 2.2 and 2.5], and it gives a characterization of the Green function of the set $E$ :

Lemma 4.1. Let $\left(\mathbb{C}_{v},|\cdot|_{v}\right)$ be either an archimedean or a nonarchimedean field. Let $E$ be a compact subset of $\mathbb{A}_{\mathrm{Berk}, \mathbb{C}_{v}}^{1}$ and $U$ the unbounded component of $\mathbb{A}_{\mathrm{Berk}, \mathbb{C}_{v}}^{1} \backslash E$.

(1) If $\gamma(E)>0$ (that is, $V(E)<\infty)$, then $G_{E}(z)=V(E)+\log |z|_{v}+o(1)$ for all $z \in \mathbb{A}_{\mathrm{Berk}, \mathbb{C}_{v}}^{1}$ such that $|z|_{v}$ is sufficiently large. Furthermore, the o(1) term may be omitted if $v$ is nonarchimedean.

(2) If $G_{E}(z)=0$ for all $z \in E$, then $G_{E}$ is continuous on $\mathbb{A}_{\text {Berk, } \mathbb{C}_{v}}^{1}, \operatorname{Supp}\left(\mu_{E}\right)=\partial U$, and $G_{E}(z)>0$ if and only if $z \in U$.

(3) If $G: \mathbb{A}_{\mathrm{Berk}, \mathbb{C}_{v}}^{1} \rightarrow \mathbb{R}$ is a continuous subharmonic function that is harmonic on $U$, identically zero on $E$, and such that $G(z)-\log ^{+}|z|_{v}$ is bounded, then $G=G_{E}$. Furthermore, if $G(z)=\log |z|_{v}+V+o(1)\left(a s|z|_{v} \rightarrow \infty\right)$ for some $V<\infty$, then $V(E)=V$, so $\gamma(E)=e^{-V}$. 
To state the equidistribution result from [Baker and Rumely 2010], we consider the compact Berkovich adelic sets, which are of the form

$$
\mathbb{E}:=\prod_{v \in \Omega} E_{v},
$$

where $E_{v}$ is a nonempty compact subset of $\mathbb{A}_{\text {Berk, } \mathbb{C}_{v}}^{1}$ for each $v \in \Omega$ and where $E_{v}$ is the closed unit disk $\mathscr{D}(0,1)$ in $\mathbb{A}_{\text {Berk, } \mathbb{C}_{v}}^{1}$ for all but finitely many $v \in \Omega$. The logarithmic capacity $\gamma(\mathbb{E})$ of $\mathbb{E}$ is defined by

$$
\gamma(\mathbb{E}):=\prod_{v \in \Omega} \gamma\left(E_{v}\right)^{N_{v}},
$$

where the positive integers $N_{v}$ are the ones associated with the product formula on the global field $K$. Note that this is a finite product since $\gamma\left(E_{v}\right)=\gamma(\mathscr{D}(0,1))=1$ for all but finitely many $v \in \Omega$. Let $G_{v}=G_{E_{v}}$ be the Green function of $E_{v}$ relative to $\infty$ for each $v \in \Omega$. For every $v \in \Omega$, we fix an embedding $\bar{K} \hookrightarrow \mathbb{C}_{v}$. Let $S \subset \bar{K}$ be any finite subset that is invariant under the action of the Galois group $\operatorname{Gal}(\bar{K} / K)$. We define the height $h_{\mathbb{E}}(S)$ of $S$ relative to $\mathbb{E}$ by

$$
h_{\mathbb{E}}(S):=\sum_{v \in \Omega} N_{v}\left(\frac{1}{|S|} \sum_{z \in S} G_{v}(z)\right) .
$$

Note that this definition is independent of any particular embedding $\bar{K} \hookrightarrow \mathbb{C}_{v}$ that we choose at $v \in \Omega$. The following is a special case of the equidistribution result [Baker and Rumely 2010, Theorem 7.52].

Theorem 4.3. Let $\mathbb{E}=\prod_{v \in \Omega} E_{v}$ be a compact Berkovich adelic set with $\gamma(\mathbb{E})=1$. Suppose that $S_{n}$ is a sequence of $\operatorname{Gal}(\bar{K} / K)$-invariant finite subsets of $\bar{K}$ with $\left|S_{n}\right| \rightarrow \infty$ and $h_{\mathbb{E}}\left(S_{n}\right) \rightarrow 0$ as $n \rightarrow \infty$. For each $v \in \Omega$ and for each $n$, let $\delta_{n}$ be the discrete probability measure supported equally on the elements of $S_{n}$. Then the sequence of measures $\left\{\delta_{n}\right\}$ converges weakly to $\mu_{v}$, the equilibrium measure on $E_{v}$.

\section{General results about the dynamics of polynomials $f_{\lambda}$}

In this section, we work with a family of polynomials $f_{\lambda}$ as given in Section 2 , that is,

$$
f_{\lambda}(x)=x^{d}+\sum_{i=0}^{d-2} c_{i}(\lambda) x^{i}
$$

with $c_{i}(\lambda) \in \mathbb{C}[\lambda]$ for $i=0, \ldots, d-2$. As before, we may rewrite our family of polynomials as

$$
f_{\lambda}(x)=P(x)+\sum_{j=1}^{r} Q_{j}(x) \cdot \lambda^{m_{j}},
$$


where $P(x)$ is a polynomial of degree $d$ in normal form, each $Q_{i}$ has degree at most equal to $d-2, r$ is a nonnegative integer, and $m_{0}:=0<m_{1}<\cdots<m_{r}$. Let $\boldsymbol{c}(\lambda) \in \mathbb{C}[\lambda]$ be given, and let $K$ be the field extension of $\mathbb{Q}$ generated by all the coefficients of $c_{i}(\lambda)$ for $i=0, \ldots, d-2$ and of $c(\lambda)$.

We define $g_{\boldsymbol{c}, n}(\lambda):=f_{\lambda}^{n}(\boldsymbol{c}(\lambda))$ for each $n \in \mathbb{N}$. Assume $m:=\operatorname{deg}(\boldsymbol{c})$ satisfies the property (ii) from Theorem 2.3 , that is,

$$
m=\operatorname{deg}(\boldsymbol{c}) \geq m_{r} .
$$

Furthermore, if $r=0$, we assume $m \geq 1$ (see also Remarks 2.4(c)). We let $q_{m}$ be the leading coefficient of $\boldsymbol{c}(\lambda)$. In the next lemma, we compute the degrees of all polynomials $g_{c, n}$ for all positive integers $n$.

Lemma 5.2. With the hypothesis above, the polynomial $g_{c, n}(\lambda)$ has degree $m \cdot d^{n}$ and leading coefficient $q_{m}^{d^{n}}$ for each $n \in \mathbb{N}$.

Proof. The assertion follows easily by induction on $n$ using (5.1) since the term of highest degree in $\lambda$ from $g_{c, n}(\lambda)$ is $c(\lambda)^{d^{n}}$.

We immediately obtain as a corollary of Lemma 5.2 the fact that $c$ is not preperiodic for $\boldsymbol{f}$. The set of all $\lambda \in \mathbb{C}$ such that $\boldsymbol{c}(\lambda)$ is preperiodic for $f_{\lambda}$ is denoted by $\operatorname{Prep}(\boldsymbol{c})$. The following result is an immediate consequence of Lemma 5.2:

Corollary 5.3. $\operatorname{Prep}(\boldsymbol{c}) \subset \bar{K}$.

\section{Capacities of generalized Mandelbrot sets}

We continue with the notation from Sections 4 and 5 . Let $c:=c(\lambda) \in \mathbb{C}[\lambda]$ be a nonconstant polynomial, and let $K$ be a product formula field containing the coefficients of each $c_{i}(\lambda)$ for $i=0, \ldots, d-2$ and of $\boldsymbol{c}$. We let $\Omega_{K}$ be the set of inequivalent absolute values of the global field $K$, and let $v \in \Omega_{K}$. Assume that $\boldsymbol{c}(\lambda)=q_{m} \lambda^{m}+$ (lower terms), where $m=\operatorname{deg}(\boldsymbol{c})$ satisfies the condition (5.1).

Our goal is to compute the logarithmic capacities of the $v$-adic generalized Mandelbrot sets $M_{\boldsymbol{c}, v}$ defined in Section 3. Following [Baker and DeMarco 2011], we extend the definition of our $v$-adic Mandelbrot set $M_{\boldsymbol{c}, v}$ to be a subset of the affine Berkovich line $A_{\mathrm{Berk}, \mathbb{C}_{v}}^{1}$ as follows:

$$
M_{\boldsymbol{c}, v}:=\left\{\lambda \in \mathbb{A}_{\text {Berk, } \mathbb{C}_{v}}^{1}: \sup _{n}\left[g_{\boldsymbol{c}, n}(T)\right]_{\lambda}<\infty\right\} .
$$

Note that if $\mathbb{C}_{v}$ is a nonarchimedean field, then our present definition for $M_{\boldsymbol{c}, v}$ yields more points than our definition from Section 3. Let $\lambda \in \mathbb{C}_{v}$, and recall the local canonical height $\hat{h}_{\lambda, v}(x)$ of $x \in \mathbb{C}_{v}$ is given by the formula

$$
\hat{h}_{\lambda, v}(x):=\hat{h}_{f_{\lambda}, v}(x)=\lim _{n \rightarrow \infty} \frac{\log ^{+}\left|f_{\lambda}^{n}(x)\right|_{v}}{d^{n}} .
$$


Notice that $\hat{h}_{\lambda, v}(x)$ is a continuous function of both $\lambda$ and $x$ (see [Branner and Hubbard 1988, Proposition 1.2] for polynomials over complex numbers; the proof for the nonarchimedean case is similar). As $\mathbb{C}_{v}$ is a dense subspace of $\mathbb{A}_{\text {Berk, }}^{1} \mathbb{C}_{v}$, continuity in $\lambda$ implies that the canonical local height function $\hat{h}_{\lambda, v}(\boldsymbol{c}(\lambda))$ has a natural extension on $\mathbb{A}_{\text {Berk, } \mathbb{C}_{v}}^{1}$. (Since the topology on $\mathbb{C}_{v}$ is the restriction of the weak topology on $\mathbb{A}_{\text {Berk, } \mathbb{C}_{v}}^{1}$, any continuous function on $\mathbb{C}_{v}$ automatically has a unique extension to $\mathbb{A}_{\text {Berk, } \mathbb{C}_{v}}$.) We will view $\hat{h}_{\lambda, v}(\boldsymbol{c}(\lambda))$ as a continuous function on $\mathbb{A}_{\text {Berk, } \mathbb{C}_{v}}^{1}$ in the following. It follows from the definition of $M_{\boldsymbol{c}, v}$ that $\lambda \in M_{\boldsymbol{c}, v}$ if and only if $\hat{h}_{\lambda, v}(\boldsymbol{c}(\lambda))=0$. Thus, $M_{\boldsymbol{c}, v}$ is a closed subset of $\mathbb{A}_{\mathrm{Berk}, \mathbb{C}_{v}}^{1}$. In fact, the following is true:

Proposition 6.1. $M_{\boldsymbol{c}, v}$ is a compact subset of $\mathbb{A}_{\mathrm{Berk}, \mathbb{C}_{v}}^{1}$.

We already showed that $M_{\boldsymbol{c}, v}$ is a closed subset of the locally compact space $\mathbb{A}_{\text {Berk, } \mathbb{C}_{v}}^{1}$, and thus, in order to prove Proposition 6.1 , we only need to show that $M_{\boldsymbol{c}, v}$ is a bounded subset of $\mathbb{A}_{\mathrm{Berk}, \mathbb{C}_{v}}^{1}$. If $\boldsymbol{f}$ is a constant family of polynomials, then Proposition 6.1 follows from our assumption that $\operatorname{deg}(\boldsymbol{c}) \geq 1$. Indeed, if $|\lambda|_{v}$ is large, then $|\boldsymbol{c}(\lambda)|_{v}$ is large, and thus, $\left|\boldsymbol{f}^{n}(\boldsymbol{c}(\lambda))\right|_{v} \rightarrow \infty$ as $n \rightarrow \infty$. Furthermore, for nonarchimedean place $v$, if $|\lambda|_{v}$ is sufficiently large, then (assuming $v$ is nonarchimedean)

$$
\left|\boldsymbol{f}^{n}(\boldsymbol{c}(\lambda))\right|_{v}=|\boldsymbol{c}(\lambda)|_{v}^{d^{n}}=\left|q_{m} \lambda^{m}\right|_{v}^{d^{n}} .
$$

So now we are left with the case that $f$ is not a constant family, that is, $r \geq 1$.

Lemma 6.3. Assume $r \geq 1$, that is, $\boldsymbol{f}$ is not a constant family of polynomials. Then $M_{c, v}$ is a bounded subset of $\mathbb{A}_{\text {Berk, } \mathbb{C}_{v}}^{1}$.

Proof. First we rewrite as before

$$
f_{\lambda}(x)=P(x)+\sum_{j=1}^{r} Q_{j}(x) \cdot \lambda^{m_{j}}
$$

with $P(x)$ in normal form of degree $d$ and each polynomial $Q_{j}$ of degree $e_{j} \leq d-2$; also, $0<m_{1}<\cdots<m_{r}$. We know $m=\operatorname{deg}(\boldsymbol{c}) \geq m_{r}$.

Since $q_{m} \lambda^{m}$ is the leading monomial in $c$, there exists a positive real number $C_{1}$ depending only on $v$, coefficients of $c_{i}(\lambda)$ for $i=0, \ldots, d-2$, and $c$ such that if $|\lambda|_{v}>C_{1}$, then $|\boldsymbol{c}(\lambda)|_{v}>\frac{1}{2}\left|q_{m}\right|_{v} \cdot|\lambda|_{v}^{m}$.

Let $\alpha:=\max _{i=1}^{r} m_{i} /\left(d-e_{i}\right)$; then $\alpha \leq m_{r} / 2$ since $e_{i} \leq d-2$ for all $i$. There exist positive real numbers $C_{2}$ and $C_{3}$ (depending only on $v$ and the coefficients of $\left.c_{i}(\lambda)\right)$ such that if $|\lambda|_{v}>C_{2}$ and $|x|_{v}>C_{3}|\lambda|_{v}^{\alpha}$, then

$$
\left|f_{\lambda}(x)\right|_{v}>\frac{1}{2}|x|_{v}^{d}>|x|_{v}
$$

and thus, $\left|f_{\lambda}^{n}(x)\right|_{v} \rightarrow \infty$ as $n \rightarrow \infty$. 
However, since $m \geq m_{r} \geq 2 \alpha>\alpha$, we conclude that if $|\lambda|_{v}>\left(2 C_{3} /\left|q_{m}\right|_{v}\right)^{1 /(m-\alpha)}$, then

$$
\frac{1}{2}\left|q_{m}\right|_{v} \cdot|\lambda|_{v}^{m}>C_{3}|\lambda|_{v}^{\alpha}
$$

We let $C_{4}:=\max \left\{C_{1}, C_{2},\left(2 C_{3} /\left|q_{m}\right|_{v}\right)^{1 /(m-\alpha)},\left|q_{m}\right|_{v}^{-1 / m}\right\}$. So if $|\lambda|_{v}>C_{4}$, then

$$
|\boldsymbol{c}(\lambda)|_{v}>\frac{1}{2}\left|q_{m}\right|_{v} \cdot|\lambda|_{v}^{m}>C_{3}|\lambda|_{v}^{\alpha},
$$

and thus, $\left|f_{\lambda}^{n}(\boldsymbol{c}(\lambda))\right|_{v} \rightarrow \infty$ as $n \rightarrow \infty$. We conclude that if $\lambda \in M_{\boldsymbol{c}, v}$, then $|\lambda|_{v} \leq C_{4}$, as desired.

Remark 6.4. It is possible to make the constants in the proof above explicit. Moreover, for a nonarchimedean place $v$, the estimate of the absolute values can be precise. For example, if $v$ is nonarchimedean, we can ensure that if $|\lambda|_{v}>C_{4}$, then

$$
\left|f_{\lambda}^{n}(\boldsymbol{c}(\lambda))\right|_{v}=\left|q_{m} \lambda^{m}\right|_{v}^{d^{n}} \text { for all } n \geq 1 .
$$

Theorem 6.6. The logarithmic capacity of $M_{\boldsymbol{c}, v}$ is $\gamma\left(M_{\boldsymbol{c}, v}\right)=\left|q_{m}\right|_{v}^{-1 / m}$.

The strategy for the proof of Theorem 6.6 is to construct a continuous subharmonic function $G_{c, v}: \mathbb{A}_{\text {Berk, } \mathbb{C}_{v}}^{1} \rightarrow \mathbb{R}$ satisfying Lemma 4.1(3). Analogously to the family $f_{\lambda}(x)=x^{d}+\lambda$ treated in [Baker and DeMarco 2011], we let

$$
G_{c, v}(\lambda):=\lim _{n \rightarrow \infty} \frac{1}{\operatorname{deg}\left(g_{c, n}\right)} \log ^{+}\left[g_{c, n}(T)\right]_{\lambda} .
$$

Then by a similar reasoning as in the proof of [ibid., Proposition 3.7], it can be shown that the limit exists for all $\lambda \in \mathbb{A}_{\text {Berk, } \mathbb{C}_{v}}^{1}$. In fact, by the definition of canonical local height, for $\lambda \in \mathbb{C}_{v}$ we have

$$
\begin{aligned}
G_{\boldsymbol{c}, v}(\lambda) & =\lim _{n \rightarrow \infty} \frac{1}{m d^{n}} \log ^{+}\left|f_{\lambda}^{n}(\boldsymbol{c}(\lambda))\right|_{v} \quad \text { since } \operatorname{deg}\left(g_{\boldsymbol{c}, n}\right)=m d^{n} \text { by Lemma 5.2, } \\
& =\frac{1}{m} \cdot \hat{h}_{f_{\lambda}, v}(\boldsymbol{c}(\lambda)) \quad \text { by the definition of canonical local height. }
\end{aligned}
$$

As a consequence of the computation above, we have the following:

Proposition 6.8 [Silverman 1994a, Theorem II.0.1; Silverman 1994b, Theorem III.0.1 and Corollary III.0.3]. We have $\hat{h}_{f_{\lambda}, v}(\boldsymbol{c}(\lambda))=\operatorname{deg}(\boldsymbol{c}) G_{\boldsymbol{c}, v}(\lambda)$.

Remark 6.9. The formula above holds in the more general case of Question 1.3; for example, one may work with a rational function $c \in \mathbb{C}(\lambda)$.

Note that $G_{\boldsymbol{c}, v}(\lambda) \geq 0$ for all $\lambda \in \mathbb{A}_{\mathrm{Berk}, \mathbb{C}_{v}}^{1}$. Moreover, we see easily that $\lambda \in M_{\boldsymbol{c}, v}$ if and only if $G_{c, v}(\lambda)=0$.

Lemma 6.10. The function $G_{\boldsymbol{c}, v}$ is the Green function for $M_{\boldsymbol{c}, v}$ relative to $\infty$.

The proof is essentially the same as the proof of [Baker and DeMarco 2011, Proposition 3.7]; we simply give a sketch of the idea. 
Proof of Lemma 6.10. We deal with the case that $v$ is nonarchimedean. (The case when $v$ is archimedean follows similarly.) So using the same argument as in the proof of [Branner and Hubbard 1988, Proposition 1.2], we observe that as a function of $\lambda$,

$$
\frac{\log ^{+}\left[g_{\boldsymbol{c}, n}(T)\right]_{\lambda}}{\operatorname{deg}\left(g_{\boldsymbol{c}, n}\right)}
$$

converges uniformly on compact subsets of $\mathbb{A}_{\mathrm{Berk}, \mathbb{C}_{v}}^{1}$. So this function is continuous and subharmonic on $\mathbb{A}_{\mathrm{Berk}, \mathbb{C}_{v}}^{1}$ and converges to $G_{\boldsymbol{c}, v}$ uniformly; hence, it follows from [Baker and Rumely 2010, Proposition 8.26(c)] that $G_{\boldsymbol{c}, v}$ is continuous and subharmonic on $\mathbb{A}_{\text {Berk, } \mathbb{C}_{v}}^{1}$. Furthermore, as remarked above, $G_{\boldsymbol{c}, v}$ is 0 on $M_{\boldsymbol{c}, v}$.

Arguing as in the proof of Lemma 6.3 (see (6.2) and (6.5)), if $|\lambda|_{v}>C_{4}$, then for $n \geq 1$ we have

$$
\left|g_{\boldsymbol{c}, n}(\lambda)\right|_{v}=\left|f_{\lambda}^{n}(\boldsymbol{c}(\lambda))\right|_{v}=\left|q_{m} \lambda^{m}\right|_{v}^{d^{n}} .
$$

Hence, for $|\lambda|_{v}>C_{4}$ we have

$$
G_{c, v}(\lambda)=\lim _{n \rightarrow \infty} \frac{1}{m d^{n}} \log \left|g_{c, n}(\lambda)\right|_{v}=\log |\lambda|_{v}+\frac{\log \left|q_{m}\right|_{v}}{m} .
$$

It follows from Lemma 4.1(3) that $G_{\boldsymbol{c}, v}$ is indeed the Green function of $M_{\boldsymbol{c}, v}$.

Now we are ready to prove Theorem 6.6.

Proof of Theorem 6.6. As in the proof of Lemma 6.10, we have

$$
G_{\boldsymbol{c}, v}(\lambda)=\log |\lambda|_{v}+\frac{\log \left|q_{m}\right|_{v}}{m}+o(1)
$$

for $|\lambda|_{v}$ sufficiently large. By Lemma 4.1(3), we find that $V\left(M_{c, v}\right)=\log \left|q_{m}\right|_{v} / m$. Hence, the logarithmic capacity of $M_{\boldsymbol{c}, v}$ is

$$
\gamma\left(M_{\boldsymbol{c}, v}\right)=e^{-V\left(M_{\boldsymbol{c}, v}\right)}=1 /\left|q_{m}\right|_{v}^{1 / m}
$$

Let $\mathbb{M}_{c}=\prod_{v \in \Omega} M_{c, v}$ be the generalized adelic Mandelbrot set associated with $c$. As a corollary to Theorem 6.6, we see $\mathbb{M}_{c}$ satisfies the hypothesis of Theorem 4.3.

Corollary 6.11. For all but finitely many nonarchimedean places $v$, we have that $M_{\boldsymbol{c}, v}$ is the closed unit disk $\mathscr{D}(0 ; 1)$ in $\mathbb{C}_{v}$; furthermore, $\gamma\left(\mathbb{M}_{\boldsymbol{c}}\right)=1$.

Proof. For each place $v$ where all coefficients of $c_{i}(\lambda)$ for $i=0, \ldots, d-2$ and of $c(\lambda)$ are $v$-adic integral and moreover $\left|q_{m}\right|_{v}=1$, we have that $M_{\boldsymbol{c}, v}=\mathscr{D}(0,1)$. Indeed, $\mathscr{D}(0,1) \subset M_{\boldsymbol{c}, v}$ since then $f_{\lambda}^{n}(\boldsymbol{c}(\lambda))$ is always a $v$-adic integer. For the converse implication, we note that each coefficient of $g_{c, n}(\lambda)$ is a $v$-adic integer while the leading coefficient is a $v$-adic unit for all $n \geq 1$; thus, $\left|g_{c, n}(\lambda)\right|_{v}=|\lambda|_{v}^{m d^{n}} \rightarrow \infty$ if $|\lambda|_{v}>1$. Note that $q_{m} \neq 0$, so the second assertion in Corollary 6.11 follows immediately by the product formula in $K$. 
Using Proposition 6.8 and the decomposition of the global canonical height as a sum of local canonical heights, we obtain the following result:

Corollary 6.12. Let $\lambda \in \bar{K}$, let $S$ be the set of $\mathrm{Gal}(\bar{K} / K)$-conjugates of $\lambda$, and let $h_{\mathbb{M}_{c}}$ be defined as in (4.2). Then $\operatorname{deg}(\boldsymbol{c}) \cdot h_{\mathbb{M}_{\boldsymbol{c}}}(\lambda)=\hat{h}_{f_{\lambda}}(\boldsymbol{c}(\lambda))$.

Remark 6.13. Let $h(\lambda)$ denote a Weil height function corresponding to the divisor $\infty$ of the parameter space that is the projective line in our case. Then it follows from [Call and Silverman 1993, Theorem 4.1] that

$$
\lim _{h(\lambda) \rightarrow \infty} \frac{\hat{h}_{f_{\lambda}}(\boldsymbol{c}(\lambda))}{h(\lambda)}=\hat{h}_{f}(\boldsymbol{c})
$$

where $\hat{h}_{f}(\boldsymbol{c})$ is the canonical height associated with the polynomial map $\boldsymbol{f}$ over the function field $\mathbb{C}(\lambda)$. Corollary 6.12 gives a precise relationship between the canonical height function on the special fiber, the height of the parameter $\lambda$, and $\hat{h}_{f}(\boldsymbol{c})$, which is equal to $\operatorname{deg}(\boldsymbol{c})$ in this case.

\section{Explicit formula for the Green function}

In this section, we work under the assumption that $|\cdot|_{v}=|\cdot|$ is archimedean, and $\mathbb{C}_{v}$ simply denotes $\mathbb{C}$ in this case. We show that in this setting we have an alternative way of representing the Green function $G_{c}:=G_{c, v}$ for the Mandelbrot set $M_{c}:=M_{c, v}$. We continue to work under the same hypothesis on $c(\lambda)$; in particular, we assume that (5.1) holds. Furthermore, if $r=0$ (that is, $f$ is a constant family of polynomials), then $m=\operatorname{deg}(\boldsymbol{c}) \geq 1$.

Since the degree in $x$ of $f_{\lambda}(x)$ is $d$, there exists a unique function $\phi_{\lambda}$ that is an analytic homeomorphism on the set $U_{R_{\lambda}}$ for some $R_{\lambda} \geq 1$ (where for any positive real number $R, U_{R}$ denotes the open set $\{z \in \mathbb{C}:|z|>R\}$ ) satisfying the following conditions:

(1) $\phi_{\lambda}$ has derivative equal to 1 at $\infty$, or more precisely, the analytic function $\psi_{\lambda}(z):=1 / \phi_{\lambda}(1 / z)$ has derivative equal to 1 at $z=0$, and

(2) $\phi_{\lambda}\left(f_{\lambda}(z)\right)=\left(\phi_{\lambda}(z)\right)^{d}$ for $|z|>R_{\lambda}$.

We can make (1) above more precise by giving the power series expansion

$$
\phi_{\lambda}(z)=z+\sum_{n=1}^{\infty} \frac{A_{\lambda, n}}{z^{n}} .
$$

From (7.1) we immediately conclude that $\left|\phi_{\lambda}(z)\right|=|z|+O_{\lambda}(1)$, and thus,

$$
\log \left|\phi_{\lambda}(z)\right|=\log |z|+O_{\lambda}(1) \quad \text { for }|z| \text { large enough. }
$$


So using that $\phi_{\lambda}\left(f_{\lambda}(z)\right)=\phi_{\lambda}(z)^{d}$, we conclude that if $|z|>R_{\lambda}$, then

$$
\lim _{n \rightarrow \infty} \frac{\log ^{+}\left|f_{\lambda}^{n}(z)\right|}{d^{n}}=\lim _{n \rightarrow \infty} \frac{\log \left|\phi_{\lambda}\left(f_{\lambda}^{n}(z)\right)\right|}{d^{n}}=\log \left|\phi_{\lambda}(z)\right| .
$$

Hence, (7.3) yields that the Green function $G^{\lambda}$ for the (filled Julia set of the) polynomial $f_{\lambda}$ equals

$$
G^{\lambda}(z):=\lim _{n \rightarrow \infty} \frac{\log \left|f_{\lambda}^{n}(z)\right|}{d^{n}}=\log \left|\phi_{\lambda}(z)\right| \quad \text { if }|z|>R_{\lambda} .
$$

For details on the Green function associated with any polynomial, see [Carleson and Gamelin 1993], where Chapter III.4 says that the function $\log \left|\phi_{\lambda}(z)\right|$ can be extended to a well-defined harmonic function on the entire basin of attraction $A_{\infty}^{\lambda}$ of the point at $\infty$ for the polynomial map $f_{\lambda}$. The set $A_{\infty}^{\lambda}$ is the complement of the filled Julia set of $f_{\lambda}$; more precisely, it is the set of all $z \in \mathbb{C}$ such that the orbit of $z$ under $f_{\lambda}$ is unbounded. Thus, on $A_{\infty}^{\lambda}$ we have

$$
G^{\lambda}(z):=\log \left|\phi_{\lambda}(z)\right|
$$

is the Green function for (the filled Julia set of) the polynomial $f_{\lambda}$. Also by [ibid., Chapter III.4], we know that

$$
R_{\lambda}:=\max _{f_{\lambda}^{\prime}(x)=0} e^{G^{\lambda}(x)} \geq 1 .
$$

In Proposition 7.6, we will show that if $|\lambda|$ is sufficiently large, then $c(\lambda)$ is in the domain of analyticity for $\phi_{\lambda}$. In particular, using (7.2) this would yield

$$
G_{c}(\lambda)=\lim _{n \rightarrow \infty} \frac{\log ^{+}\left|f_{\lambda}^{n}(\boldsymbol{c}(\lambda))\right|}{m d^{n}}=\frac{\log \left|\phi_{\lambda}(\boldsymbol{c}(\lambda))\right|}{m}=\frac{G^{\lambda}(\boldsymbol{c}(\lambda))}{m}
$$

for $|\lambda|$ sufficiently large.

Proposition 7.6. There exists a positive constant $C_{0}$ such that if $|\lambda|>C_{0}$, then $\boldsymbol{c}(\lambda)$ belongs to the analyticity domain of $\phi_{\lambda}$.

Proof. The proof is similar to that of [Baker and DeMarco 2011, Lemma 3.2]. If $\boldsymbol{f}$ is a constant family of polynomials, then the conclusion is immediate since $R_{\lambda}$ is constant (independent of $\lambda$ ), and thus, for $|\lambda|$ sufficiently large, clearly $|c(\lambda)|>R_{\lambda}$. So from now on assume $\boldsymbol{f}$ is not a constant family of polynomials, which in particular yields that $r \geq 1$ and $0<m_{1}<\cdots<m_{r}$.

First we recall that

$$
R_{\lambda}=e^{G^{\lambda}\left(x_{0}\right)}:=\max _{f_{\lambda}^{\prime}(x)=0} e^{G^{\lambda}(x)} .
$$

Next we show that $R_{\lambda} \rightarrow \infty$ as $|\lambda| \rightarrow \infty$, which will be used later in our proof.

Lemma 7.7. As $|\lambda| \rightarrow \infty$, we have $R_{\lambda} \rightarrow \infty$. 
Proof. We recall that

$$
f_{\lambda}(x)=P(x)+\sum_{i=1}^{r} \lambda^{m_{i}} \cdot Q_{i}(x),
$$

where $P(x)$ is a polynomial in normal form of degree $d$ and $0<m_{1}<\cdots<m_{r}$ are positive integers while the $Q_{i}$ are nonzero polynomials of degrees $e_{i} \leq d-2$. We have two cases.

Case 1. Each $Q_{i}(x)$ is a constant polynomial. Then the critical points of $f_{\lambda}$ are independent of $\lambda$, that is, $x_{0}=O(1)$. We let $x_{1} \in \mathbb{C}$ such that $f_{\lambda}\left(x_{1}\right)=x_{0}$. Since each $Q_{i}$ is a nonzero constant polynomial, we immediately conclude that $\left|x_{1}\right| \gg|\lambda|^{m_{r} / d}$. On the other hand, since $U_{2 R_{\lambda}} \subset \phi_{\lambda}^{-1}\left(U_{R_{\lambda}}\right)$ [Branner and Hubbard 1988, Corollary 3.3], we conclude that $\left|x_{1}\right| \leq 2 R_{\lambda}$, so $R_{\lambda} \gg|\lambda|^{m_{r} / d}$. Indeed, if $\left|x_{1}\right|>2 R_{\lambda}$, then there exists $z_{1} \in U_{R_{\lambda}}$ such that $\phi_{\lambda}^{-1}\left(z_{1}\right)=x_{1}$. Using the fact that $\phi_{\lambda}$ is a conjugacy map at $\infty$ for $f_{\lambda}$, we would obtain that

$$
x_{0}=f_{\lambda}\left(x_{1}\right)=f_{\lambda}\left(\phi_{\lambda}^{-1}\left(z_{1}\right)\right)=\phi_{\lambda}^{-1}\left(z_{1}^{d}\right) \in U_{R_{\lambda}},
$$

which contradicts the fact that $x_{0}$ is not in the analyticity domain of $\phi_{\lambda}$.

Case 2. There exists $i=1, \ldots, r$ such that $Q_{i}(x)$ is not a constant polynomial. Then the critical points of $f_{\lambda}$ vary with $\lambda$. In particular, there exists a critical point $x_{\lambda}$ of maximum absolute value such that $\left|x_{\lambda}\right| \gg|\lambda|^{m_{j} /\left(d-e_{j}\right)}$ (for some $j=1, \ldots, r$ ), where for each $i=1, \ldots, r$, we have $e_{i}=\operatorname{deg}\left(Q_{i}\right) \leq d-2$. Now, $x_{\lambda}$ is not in the domain of analyticity of $\phi_{\lambda}$, and thus, $\left|x_{\lambda}\right| \leq R_{\lambda}$, which again shows that $R_{\lambda} \rightarrow \infty$ as $|\lambda| \rightarrow \infty$.

Using that $R_{\lambda} \rightarrow \infty$, we will finish our proof. First we note that

$$
\left|\phi_{\lambda}\left(f_{\lambda}\left(x_{0}\right)\right)\right|=e^{G^{\lambda}\left(f_{\lambda}\left(x_{0}\right)\right)}=e^{d G^{\lambda}\left(x_{0}\right)}=R_{\lambda}^{d} .
$$

Note that $\phi_{\lambda}(z)$ is analytic on $U_{R_{\lambda}}$ while $\log \left|\phi_{\lambda}(z)\right|$ is continuous for $|z| \geq R_{\lambda}$. Moreover, whenever it is defined, $G^{\lambda}\left(f_{\lambda}(z)\right)=d G^{\lambda}(z)$, so also using (7.4), we obtain (7.8).

Now for $|\lambda|$ sufficiently large, we have that $R_{\lambda}^{d} / 2>R_{\lambda}$ (since $R_{\lambda} \rightarrow \infty$ according to Lemma 7.7). So $U_{R_{\lambda}^{d}} \subset \phi_{\lambda}\left(U_{R_{\lambda}^{d} / 2}\right)$ [Branner and Hubbard 1988, Corollary 3.3], and thus,

$$
\left|f_{\lambda}\left(x_{0}\right)\right| \geq \frac{1}{2} R_{\lambda}^{d}
$$

Case 1. We have $\operatorname{deg}\left(Q_{i}\right)=0$ for each $i$. Then $x_{0}=O(1)$ as noticed in Lemma 7.7, and thus, using (7.9) we obtain that $|\lambda|^{m_{r}} \gg R_{\lambda}^{d}$. Since $\operatorname{deg}(\boldsymbol{c})=m \geq m_{r}$, we obtain

$$
|c(\lambda)| \geq\left|q_{m}\right| \cdot|\lambda|^{m}-\left|O\left(\lambda^{m-1}\right)\right| \gg R_{\lambda}^{d}>R_{\lambda}
$$

if $|\lambda|$ is sufficiently large. 
Case 2. If not all of the $Q_{i}$ are constant polynomials, then we still know that

$$
\left|x_{0}\right| \ll|\lambda|^{\max _{i=1}^{r} m_{i} /\left(d-e_{i}\right)} \ll|\lambda|^{m_{r} / 2}
$$

because $e_{i} \leq d-2$ for each $i$. Therefore,

$$
R_{\lambda}^{d} \ll\left|f_{\lambda}\left(x_{0}\right)\right| \ll|\lambda|^{d m_{r} / 2}
$$

On the other hand, $|\boldsymbol{c}(\lambda)| \sim|\lambda|^{m}$ and $m \geq m_{r}$, which yield that

$$
|c(\lambda)| \gg|\lambda|^{m} \gg R_{\lambda}^{2} \gg R_{\lambda}
$$

by (7.10). This concludes the proof of Proposition 7.6.

Therefore, for large $|\lambda|$, the point $c(\lambda)$ is in the domain of analyticity for $\phi_{\lambda}$, which allows us to conclude that (7.5) holds.

We know from [Carleson and Gamelin 1993] that for each $\lambda \in \mathbb{C}$ and for each $z \in \mathbb{C}$ sufficiently large in absolute value, we have

$$
\phi_{\lambda}(z)=z \prod_{n=0}^{\infty}\left(\frac{f_{\lambda}^{n+1}(z)}{f_{\lambda}^{n}(z)^{d}}\right)^{1 / d^{n+1}}
$$

and thus,

$$
\phi_{\lambda}(z)=z \prod_{n=0}^{\infty}\left(1+\frac{Q_{0}\left(f_{\lambda}^{n}(z)\right)+\sum_{i=1}^{r} Q_{i}\left(f_{\lambda}^{n}(z)\right) \cdot \lambda^{m_{i}}}{f_{\lambda}^{n}(z)^{d}}\right)^{1 / d^{n+1}}
$$

where $Q_{0}(x):=P(x)-x^{d}$ is a polynomial of degree at most equal to $d-2$. We showed in Proposition 7.6 that $\phi_{\lambda}(\boldsymbol{c}(\lambda))$ is well-defined; furthermore, the function $\phi_{\lambda}(\boldsymbol{c}(\lambda)) / \boldsymbol{c}(\lambda)$ can be expressed near $\infty$ as the infinite product above. Indeed, for each $n \in \mathbb{N}$, the order of magnitude of the numerator in the $n$-th fraction from the product appearing in (7.12) when we substitute $z=\boldsymbol{c}(\lambda)$ is at most

$$
|\lambda|^{m+(d-2) m d^{n}} \leq|\lambda|^{m(d-1) d^{n}}
$$

while the order of magnitude of the denominator is $|\lambda|^{m d^{n+1}}$. This guarantees the convergence of the product from (7.12) corresponding to $\phi_{\lambda}(\boldsymbol{c}(\lambda)) / \boldsymbol{c}(\lambda)$. We conclude that

$$
\begin{aligned}
& \phi_{\lambda}(\boldsymbol{c}(\lambda)) \text { is an analytic function of } \lambda \text { (for large } \lambda \text { ) and moreover } \\
& \phi_{\lambda}(\boldsymbol{c}(\lambda))=q_{m} \lambda^{m}+O\left(\lambda^{m-1}\right)
\end{aligned}
$$




\section{Proof of Theorem 2.3: Algebraic case}

We work under the hypothesis of Theorem 2.3, and we continue with the notation from the previous sections. Furthermore, we prove Theorem 2.3 under the extra assumptions that

$$
\boldsymbol{a}, \boldsymbol{b} \in \overline{\mathbb{Q}}[\lambda] \quad \text { and } \quad c_{i} \in \overline{\mathbb{Q}}[\lambda] \text { for each } i=0, \ldots, d-2 .
$$

Recall that $f_{\lambda}(x)=x^{d}+\sum_{i=0}^{d-2} c_{i}(\lambda) x^{i}$, where we require that $c_{i} \in \overline{\mathbb{Q}}[\lambda]$ for $i=0, \ldots, d-2$. Let $\boldsymbol{a}, \boldsymbol{b} \in \overline{\mathbb{Q}}[\lambda]$ satisfy the hypotheses (i) and (ii) of Theorem 2.3. Let $K$ be the number field generated by the coefficients of $c_{i}(\lambda)$ for $i=0, \ldots, d-2$ and of $\boldsymbol{a}(\lambda)$ and $\boldsymbol{b}(\lambda)$. Let $\Omega_{K}$ be the set of all inequivalent absolute values on $K$.

Next, assume there exist infinitely many $\lambda$ such that both $\boldsymbol{a}(\lambda)$ and $\boldsymbol{b}(\lambda)$ are preperiodic for $f_{\lambda}$. At the expense of replacing $\boldsymbol{a}(\lambda)$ by $f_{\lambda}^{k}(\boldsymbol{a}(\lambda))$ and $\boldsymbol{b}(\lambda)$ by $f_{\lambda}^{\ell}(\boldsymbol{b}(\lambda))$, we may assume that the polynomials

$\boldsymbol{a}(\lambda)$ and $\boldsymbol{b}(\lambda)$ have the same leading coefficient and degree $m \geq m_{r}$.

Let $h_{\mathbb{M}_{a}}(z)$ and $h_{\mathbb{M}_{b}}(z)$ be the heights of $z \in \bar{K}$ relative to the adelic generalized Mandelbrot sets $\mathbb{M}_{a}:=\prod_{v \in \Omega_{K}} M_{a, v}$ and $\mathbb{M}_{b}$ as defined in Section 6. Note that if $\lambda \in \bar{K}$ is a parameter such that $\boldsymbol{a}(\lambda)$ and $\boldsymbol{b}(\lambda)$ are preperiodic for $f_{\lambda}$, then $h_{\mathbb{M}_{a}}(\lambda)=0$ by Corollary 6.12. So we may apply the equidistribution result from [Baker and Rumely 2010, Theorem 7.52] (see our Theorem 4.3) and conclude that $M_{\boldsymbol{a}, v}=M_{\boldsymbol{b}, v}$ for each place $v \in \Omega_{K}$. Indeed, we know that there exists an infinite sequence $\left\{\lambda_{n}\right\}_{n \in \mathbb{N}}$ of distinct numbers $\lambda \in \bar{K}$ such that both $\boldsymbol{a}(\lambda)$ and $\boldsymbol{b}(\lambda)$ are preperiodic for $f_{\lambda}$. So for each $n \in \mathbb{N}$, we may take $S_{n}$ to be the union of the sets of Galois conjugates for $\lambda_{m}$ for all $1 \leq m \leq n$. Clearly, $\# S_{n} \rightarrow \infty$ as $n \rightarrow \infty$, and also each $S_{n}$ is $\operatorname{Gal}(\bar{K} / K)$-invariant. Finally, $h_{\mathbb{M}_{a}}\left(S_{n}\right)=h_{\mathbb{M}_{b}}\left(S_{n}\right)=0$ for all $n \in \mathbb{N}$, and thus, Theorem 4.3 applies in this case. We obtain that $\mu_{\mathbb{M}_{a}}=\mu_{\mathbb{M}_{b}}$, and since they are both supported on $\mathbb{M}_{a}$ and $\mathbb{M}_{b}$, respectively, we also get that $\mathbb{M}_{\boldsymbol{a}}=\mathbb{M}_{\boldsymbol{b}}$. The following lemma applies in the generality of Theorem 2.3, and it will finish our proof. (Note that since $K$ is a number field, it has at least one archimedean valuation.)

Lemma 8.3. Let $\boldsymbol{f}, \boldsymbol{a}$, and $\boldsymbol{b}$ be as in Theorem 2.3; in particular, assume they are all defined over $\mathbb{C}$. Let $|\cdot|$ be the usual archimedean absolute value on $\mathbb{C}$, and let $M_{a}$ and $M_{b}$ be the corresponding complex Mandelbrot sets. If $M_{a}=M_{b}$, then $\boldsymbol{a}=\boldsymbol{b}$.

Proof. Since $M_{a}=M_{b}$, then the corresponding Green functions are also the same, that is, (using (7.5) and (8.2))

$$
\left|\phi_{\lambda}(\boldsymbol{a}(\lambda))\right|=\left|\phi_{\lambda}(\boldsymbol{b}(\lambda))\right| \text { for all }|\lambda| \text { sufficiently large. }
$$

On the other hand, for $|z|$ large, the function $h(z):=\phi_{z}(\boldsymbol{a}(z)) / \phi_{z}(\boldsymbol{b}(z))$ is an analytic function of constant absolute value. (Note that the denominator does not vanish 
since $\phi_{\lambda}$ is a homeomorphism for a neighborhood of $\infty$.) By the open mapping theorem, we conclude that $h(z):=u$ is a constant (for some $u \in \mathbb{C}$ of absolute value equal to 1 ); that is,

$$
\phi_{\lambda}(\boldsymbol{a}(\lambda))=u \cdot \phi_{\lambda}(\boldsymbol{b}(\lambda)) .
$$

Using (7.13) and (7.14) (also note that $\boldsymbol{a}(\lambda)$ and $\boldsymbol{b}(\lambda)$ have the same leading coefficient), we have $u=1$. Using that $\phi_{\lambda}$ is a homeomorphism on a neighborhood of the infinity, we conclude that $\boldsymbol{a}(\lambda)=\boldsymbol{b}(\lambda)$ for $\lambda$ sufficiently large in absolute value and thus for all $\lambda$ as desired. (Note that $\boldsymbol{a}$ and $\boldsymbol{b}$ are polynomials.)

Remark 8.5. Our proof (similar to the proof from [Baker and DeMarco 2011]) only uses in an essential way the information that $M_{a}=M_{b}$, that is, that the Mandelbrot sets over the complex numbers corresponding to $\boldsymbol{a}$ and $\boldsymbol{b}$ are equal, even though we know that $M_{\boldsymbol{a}, v}=M_{\boldsymbol{b}, v}$ for all places $v$.

\section{Proof of Theorem 2.3: The converse implication}

Now we prove the converse implication in Theorem 2.3 in the general case, that is, for polynomials $c_{0}, \ldots, c_{d-2}, \boldsymbol{a}$, and $\boldsymbol{b}$ with arbitrary complex coefficients. Again at the expense of replacing $\boldsymbol{a}(\lambda)$ by $f_{\lambda}^{k}(\boldsymbol{a}(\lambda))$ and replacing $\boldsymbol{b}(\lambda)$ by $f_{\lambda}^{\ell}(\boldsymbol{b}(\lambda))$, we may assume $\boldsymbol{a}(\lambda)=\boldsymbol{b}(\lambda)$. The following result will finish the converse statement in Theorem 2.3:

Proposition 9.1. Let $\boldsymbol{c} \in \mathbb{C}[\lambda]$ of degree $m \geq m_{r}$. Let $\operatorname{Prep}(\boldsymbol{c})$ be the set consisting of all $\lambda \in \mathbb{C}$ such that $\boldsymbol{c}(\lambda)$ is preperiodic under $f_{\lambda}$, and let $M_{c}$ be the set of all $\lambda \in \mathbb{C}$ such that the orbit of $\boldsymbol{c}(\lambda)$ under the action of $f_{\lambda}$ is bounded with respect to the usual archimedean metric on $\mathbb{C}$. Then the closure in $\mathbb{C}$ of the set $\operatorname{Prep}(\boldsymbol{c})$ contains $\partial M_{c}$. In particular, $\operatorname{Prep}(\boldsymbol{c})$ is infinite.

Proof. We first claim that the equation $f_{z}(\boldsymbol{c}(z))=\boldsymbol{c}(z)$ has only finitely many solutions. Indeed, according to Lemma 5.2, the degree in $z$ of $f_{z}(\boldsymbol{c}(z))-\boldsymbol{c}(z)$ is $d m$, which means that there are at most $d m$ solutions $z \in \mathbb{C}$ for the equation $f_{z}(\boldsymbol{c}(z))=\boldsymbol{c}(z)$.

Let $x_{0} \in \partial M_{c}$, which is not a solution $z$ to $f_{z}(c(z))=c(z)$; we will show that $x_{0}$ is contained in the closure in $\mathbb{C}$ of $\operatorname{Prep}(\boldsymbol{c})$. Since we already know that if $f_{z}(\boldsymbol{c}(z))=\boldsymbol{c}(z)$, then $z \in \operatorname{Prep}(\boldsymbol{c})$, we will be done once we prove that each open neighborhood $U$ of $x_{0}$ contains at least one point from $\operatorname{Prep}(\boldsymbol{c})$.

Now, let $U$ be an open neighborhood of $x_{0}$, and let $h_{i}: U \rightarrow \mathbb{P}^{1}(\mathbb{C})$ for $i=1,2,3$ be three analytic functions with values taken in the compact Riemann sphere, given by

$$
h_{1}(z):=\infty, \quad h_{2}(z):=c(z), \quad \text { and } \quad h_{3}(z):=g_{c, 1}(z)=f_{z}(\boldsymbol{c}(z)) .
$$

Furthermore, since $x_{0}$ is not a solution for the equation $h_{2}(z)=h_{3}(z)$, then we may assume (at the expense of replacing $U$ with a smaller neighborhood of $x_{0}$ ) that the 
closures of $h_{2}(U)$ and $h_{3}(U)$ are disjoint. Therefore, the closures of $h_{1}(U), h_{2}(U)$, and $h_{3}(U)$ in $\mathbb{P}^{1}(\mathbb{C})$ are all disjoint.

As before, we let $\left\{g_{c, n}\right\}_{n \geq 2}$ be the set of polynomials $g_{c, n}(z):=f_{z}^{n}(\boldsymbol{c}(z))$. Since $x_{0} \in \partial M_{c}$, the family of analytic maps $\left\{g_{c, n}\right\}_{n \geq 2}$ is not normal on $U$. Therefore, by Montel's theorem [Beardon 1991, Theorem 3.3.6], there exists $n \geq 2$ and $z \in U$ such that $g_{\boldsymbol{c}, n}(z)=\boldsymbol{c}(z)$ or $g_{\boldsymbol{c}, n}(z)=f_{z}(\boldsymbol{c}(z))$. (Clearly, it cannot happen that $g_{c, n}(z)=\infty$.) Either way, we obtain that $z \in \operatorname{Prep}(\boldsymbol{c})$ as desired.

Since $\gamma\left(M_{c}\right)>0$, we know that $M_{c}$ is an uncountable subset of $\mathbb{C}$, and thus, its boundary is infinite; hence, $\operatorname{Prep}(\boldsymbol{c})$ is also infinite.

\section{Proof of Theorem 2.3: General case}

In this section, we finish the proof of Theorem 2.3. With the same notation as in Theorem 2.3, we replace $\boldsymbol{a}$ and $\boldsymbol{b}$ with $f_{\lambda}^{k}(\boldsymbol{a}(\lambda))$ and $f_{\lambda}^{\ell}(\boldsymbol{b}(\lambda))$, respectively; thus, $\boldsymbol{a}(\lambda)$ and $\boldsymbol{b}(\lambda)$ are polynomials with the same degree and same leading coefficient. We assume there exist infinitely many $\lambda \in \mathbb{C}$ such that both $\boldsymbol{a}(\lambda)$ and $\boldsymbol{b}(\lambda)$ are preperiodic for $f_{\lambda}$; we will prove that $\boldsymbol{a}=\boldsymbol{b}$.

Let $K$ denote the field generated over $\overline{\mathbb{Q}}$ by adjoining the coefficients of each $c_{i}$ (for $i=1, \ldots, d-2$ ) and adjoining the coefficients of $\boldsymbol{a}$ and of $\boldsymbol{b}$. According to Corollary 5.3, if there exists $\lambda \in \mathbb{C}$ such that $\boldsymbol{a}(\lambda)($ or $\boldsymbol{b}(\lambda))$ is preperiodic for $f_{\lambda}$, then $\lambda \in \bar{K}$, where $\bar{K}$ denotes the algebraic closure of $K$ in $\mathbb{C}$. Let $\Omega_{K}$ be the set of inequivalent absolute values of $K$ corresponding to the divisors of a projective $\overline{\mathbb{Q}}$ variety $\mathscr{V}$ regular in codimension 1 ; then the places in $\Omega_{K}$ satisfy a product formula.

As in Section 8, we let $h_{\mathbb{M}_{a}}(z)$ and $h_{\mathbb{M}_{b}}(z)$ be the heights of $z \in \bar{K}$ relative to the adelic generalized Mandelbrot sets $\mathbb{M}_{\boldsymbol{a}}=\prod_{v \in \Omega_{K}} \boldsymbol{M}_{\boldsymbol{a}, v}$ and $\mathbb{M}_{\boldsymbol{b}}$ as defined in Section 6. Note that if $\lambda \in \bar{K}$ is a parameter such that $\boldsymbol{a}(\lambda)$ is preperiodic for $f_{\lambda}$, then $h_{\mathbb{M}_{a}}(\lambda)=0$ and $h_{\mathbb{M}_{b}}(\lambda)=0$, respectively, by Corollary 6.12 again. So arguing as in Section 8, we may apply the equidistribution result from [Baker and Rumely 2010, Theorem 7.52] (Theorem 4.3) and conclude that $\boldsymbol{M}_{\boldsymbol{a}, v}=\boldsymbol{M}_{\boldsymbol{b}, v}$ for each place $v \in \Omega_{K}$.

As observed in our proof from Section 8 (see Remark 8.5), in order to finish the proof of Theorem 2.3, it suffices to prove that $M_{a}=M_{b}$, where $M_{a}$ and $M_{b}$ are the complex Mandelbrot sets corresponding to $\boldsymbol{a}$ and $\boldsymbol{b}$, respectively. By complex Mandelbrot sets $M_{a}$ and $M_{b}$, we mean the Mandelbrot sets corresponding to $a$ and $\boldsymbol{b}$ constructed with respect to the usual archimedean metric on $\mathbb{C}$.

As before, $\operatorname{Prep}(\boldsymbol{a})$ and $\operatorname{Prep}(\boldsymbol{b})$ denote the sets of all $\lambda \in \mathbb{C}$ such that $\boldsymbol{a}(\lambda)$ and $\boldsymbol{b}(\lambda)$, respectively, are preperiodic for $f_{\lambda}$. As proved in Corollary 5.3, we know that both $\operatorname{Prep}(\boldsymbol{a})$ and $\operatorname{Prep}(\boldsymbol{b})$ are subsets of $\bar{K}$. In order to prove that $M_{a}=M_{b}$, it suffices to prove that $\operatorname{Prep}(\boldsymbol{a})$ differs from $\operatorname{Prep}(\boldsymbol{b})$ in at most finitely many points. 
To ease the notation, we define the symmetric difference of $\operatorname{Prep}(\boldsymbol{a})$ and $\operatorname{Prep}(\boldsymbol{b})$ as

$$
\operatorname{PrepDiff}(\boldsymbol{a}, \boldsymbol{b}):=(\operatorname{Prep}(\boldsymbol{a}) \backslash \operatorname{Prep}(\boldsymbol{b})) \cup(\operatorname{Prep}(\boldsymbol{b}) \backslash \operatorname{Prep}(\boldsymbol{a})) .
$$

Proposition 10.1. If the set $\operatorname{PrepDiff}(\boldsymbol{a}, \boldsymbol{b})$ is finite, then $M_{a}=M_{b}$.

Proof. Since $M_{a}$ contains all points $\lambda \in \mathbb{C}$ such that $\lim _{n \rightarrow \infty} \log ^{+}\left|f_{\lambda}^{n}(a)\right| / d^{n}=0$, the maximum modulus principle yields that the complement of $M_{a}$ in $\mathbb{C}$ is connected; that is, $M_{a}$ is a full subset of $\mathbb{C}$; see also [Baker and DeMarco 2011]. So both $M_{a}$ and $M_{b}$ are full subsets of $\mathbb{C}$ containing the sets $\operatorname{Prep}(\boldsymbol{a})$ and $\operatorname{Prep}(\boldsymbol{b})$ whose closures contain the boundary of $M_{a}$ and $M_{b}$, respectively (according to Proposition 9.1). As $\operatorname{Prep}(\boldsymbol{a})$ and $\operatorname{Prep}(\boldsymbol{b})$ differ by at most finitely many elements, we conclude that $M_{a}=M_{b}$.

To prove that $\operatorname{Prep}(\boldsymbol{a})$ and $\operatorname{Prep}(\boldsymbol{b})$ differ by at most finitely many elements, we observe first that if $\lambda \in \operatorname{Prep}(\boldsymbol{a})$, then $\hat{h}_{f_{\lambda}}(\boldsymbol{a}(\lambda))=0$, and thus, $\lambda^{\sigma} \in M_{\boldsymbol{a}, v}$ for all $v$ and all $\sigma \in \operatorname{Gal}(\bar{K} / K)$. (See (3.3); note that $\boldsymbol{a}(\lambda)^{\sigma}=\boldsymbol{a}\left(\lambda^{\sigma}\right)$ since $\boldsymbol{a} \in K[x]$.) Similarly, if $\lambda \in \operatorname{Prep}(\boldsymbol{b})$, then $\lambda^{\sigma} \in M_{\boldsymbol{b}, v}$ for each place $v \in \Omega_{K}$ and each Galois morphism $\sigma$. We would like to use the reverse implication, that is, characterize the elements $\operatorname{Prep}(\boldsymbol{a})$ as the set of all $\lambda \in \bar{K}$ such that $\lambda^{\sigma} \in M_{\boldsymbol{a}, v}$ for each place $v$ and for each Galois morphism $\sigma$. This is true if $f_{\lambda}$ is not isotrivial over $\overline{\mathbb{Q}}$ by Benedetto's result [2005]. In this case, $\operatorname{Prep}(\boldsymbol{a})$ and $\operatorname{Prep}(\boldsymbol{b})$ are exactly the sets of $\lambda \in \bar{K}$ such that $h_{\mathbb{M}_{a}}(\lambda)=0$ and $h_{\mathbb{M}_{a}}(\lambda)=0$, respectively. However, notice that if $f_{\lambda} \in \overline{\mathbb{Q}}[x]$, then

$\lambda^{\sigma} \in M_{a, v}$ for all $v \in \Omega_{K}$ and $\sigma \in \operatorname{Gal}(\bar{K} / K)$ if and only if $\boldsymbol{a}(\lambda) \in \overline{\mathbb{Q}}$.

We see that in this case, $\operatorname{Prep}(\boldsymbol{a})$ is strictly smaller than the set of $\lambda \in \bar{K}$ such that $h_{\mathbb{M}_{a}}(\lambda)=0$. So we will prove that $\operatorname{Prep}(\boldsymbol{a})$ and $\operatorname{Prep}(\boldsymbol{b})$ differ by at most finitely many elements by splitting our analysis into two cases depending on whether there exist infinitely many $\lambda \in \mathbb{C}$ such that $f_{\lambda}$ is conjugate to a polynomial with coefficients in $\overline{\mathbb{Q}}$. The following easy result is key for our argument:

Lemma 10.2. For any $\lambda \in \mathbb{C}$, the polynomial $f_{\lambda}(x)$ is conjugate to a polynomial with coefficients in $\overline{\mathbb{Q}}$ if and only if $c_{i}(\lambda) \in \overline{\mathbb{Q}}$ for each $i=1, \ldots, d-2$.

Proof. One direction is obvious. Now, assume $f_{\lambda}$ is conjugate to a polynomial with coefficients in $\overline{\mathbb{Q}}$. Let $\delta(x):=a x+b$ be a linear polynomial so $\delta^{-1} \circ f_{\lambda} \circ \delta \in \overline{\mathbb{Q}}[x]$. Since $f_{\lambda}$ is in normal form, we note that $a, b \in \overline{\mathbb{Q}}$ for otherwise the leading coefficient or the next-to-leading coefficient is not algebraic. Now, it is clear that each $c_{i}(\lambda) \in \overline{\mathbb{Q}}$ as desired.

Let $S$ be the set of all $\lambda \in \mathbb{C}$ such that $f_{\lambda}$ is conjugate to a polynomial in $\overline{\mathbb{Q}}[x]$. Using Lemma 10.2, $S \subset \bar{K}$ since each polynomial $c_{i}$ has coefficients in $K$ and $\overline{\mathbb{Q}} \subset K$. Also, $S$ is $\operatorname{Gal}(\bar{K} / K)$-invariant since each coefficient of each $c_{i}$ is in $K$.

Proposition 10.3. $\operatorname{PrepDiff}(\boldsymbol{a}, \boldsymbol{b}) \subset S$. 
Proof. Let $\lambda \in \bar{K} \backslash S$. Since $f_{\lambda}$ is not conjugate to a polynomial in $\overline{\mathbb{Q}}$, using Benedetto's result (see also (3.3)) we obtain that $\boldsymbol{a}(\lambda)$ is preperiodic for $f_{\lambda}$ if and only if for each $v \in \Omega_{K}$ and $\sigma \in \operatorname{Gal}(\bar{K} / K)$, the local canonical height of $\boldsymbol{a}(\lambda)^{\sigma}=\boldsymbol{a}\left(\lambda^{\sigma}\right)$ computed with respect to $f_{\lambda}^{\sigma}$ equals 0 . Since each coefficient of $c_{i}(\lambda)$ is defined over $K$, we get that $f_{\lambda}^{\sigma}=f_{\lambda^{\sigma}}$. Therefore, for each $\lambda \in \bar{K} \backslash S$, we see that $\boldsymbol{a}(\lambda)$ or $\boldsymbol{b}(\lambda)$ is preperiodic for $f_{\lambda}$ if and only if for all $v \in \Omega_{K}$ and all $\sigma \in \operatorname{Gal}(\bar{K} / K)$, we have $\lambda^{\sigma} \in M_{\boldsymbol{a}, v}$ or $\lambda^{\sigma} \in M_{\boldsymbol{b}, v}$, respectively. Using the fact that $M_{a, v}=M_{b, v}$ for all $v \in \Omega_{K}$, we conclude that if $\lambda \in \bar{K} \backslash S$, then $\lambda \in \operatorname{Prep}(a)$ if and only if $\lambda \in \operatorname{Prep}(b)$. Hence, $\operatorname{PrepDiff}(\boldsymbol{a}, \boldsymbol{b}) \subset S$ as desired.

Lemma 10.4. If $\lambda \in S$ and $\boldsymbol{a}(\lambda) \notin \overline{\mathbb{Q}}$, then $\boldsymbol{a}(\lambda)$ is not preperiodic for $f_{\lambda}$.

Proof. The assertion is immediate since for $\lambda \in S$ we have $f_{\lambda} \in \overline{\mathbb{Q}}[x]$ by the definition of $S$ (see also Lemma 10.2); hence, the set of preperiodic points of $f_{\lambda}$ is contained in $\overline{\mathbb{Q}}$. By assumption $\boldsymbol{a}(\lambda) \notin \overline{\mathbb{Q}}$; therefore, $\boldsymbol{a}(\lambda)$ is not preperiodic for $f_{\lambda}$.

Proposition 10.5. The set $\operatorname{PrepDiff}(\boldsymbol{a}, \boldsymbol{b})$ is finite.

Proof. If $S$ is a finite set, then the assertion follows from Proposition 10.3. So in the remaining part of the proof, we assume that $S$ is an infinite set. By Lemma 10.2 we know that there exist infinitely many $\lambda \in \bar{K}$ such that $c_{i}(\lambda) \in \overline{\mathbb{Q}}$ for each $i=0, \ldots, d-2$. The following lemma will be key for our proof:

Lemma 10.6. Let $L_{1} \subset L_{2}$ be algebraically closed fields of characteristic 0 , and let $f_{1}, \ldots, f_{n} \in L_{2}[x]$. If there exist infinitely many $z \in L_{2}$ such that $f_{i}(z) \in L_{1}$ for each $i=1, \ldots, n$, then there exists $h \in L_{2}[x]$, and there exist $g_{1}, \ldots, g_{n} \in L_{1}[x]$ such that $f_{i}=g_{i} \circ$ hor each $i=1, \ldots, n$.

Proof. Let $C \subset \mathbb{A}^{n}$ be the Zariski closure of the set

$$
\left\{\left(f_{1}(z), \ldots, f_{n}(z)\right): z \in L_{2}\right\} .
$$

Then $C$ is a rational curve that (by our hypothesis) contains infinitely many points over $L_{1}$. Therefore, $C$ is defined over $L_{1}$, and thus, it has a rational parametrization over $L_{1}$. Let

$$
\left(g_{1}, \ldots, g_{n}\right): \mathbb{A}^{1} \rightarrow C
$$

be a birational morphism defined over $L_{1}$; we denote by $\psi: C \rightarrow \mathbb{A}^{1}$ its inverse. (For more details, see [Shafarevich 1994, Chapter 1]). Since the closure of $C$ in $\mathbb{P}^{n}$ (by considering the usual embedding of $\mathbb{A}^{n} \subset \mathbb{P}^{n}$ ) has only one point at infinity (due to the parametrization (10.7) of $C$ ), we conclude that (perhaps after a change of coordinates) we may assume each $g_{i}$ is also a polynomial; more precisely, $g_{i} \in L_{1}[x]$. We let $h: \mathbb{A}^{1} \rightarrow \mathbb{A}^{1}$ be the rational map (defined over $L_{2}$ ) given by the composition

$$
h:=\psi \circ\left(f_{1}, \ldots, f_{n}\right) .
$$


Therefore, for each $i=1, \ldots, n$, we have $f_{i}=g_{i} \circ h$, and since both $f_{i}$ and $g_{i}$ are polynomials, we conclude that $h$ is also a polynomial, as desired.

As an immediate consequence of Lemma 10.6, we have the following result:

Corollary 10.8. Let $L_{1} \subset L_{2}$ be algebraically closed fields of characteristic 0 , and let $f_{1}, \ldots, f_{n} \in L_{2}[x]$. If there exist infinitely many $z \in L_{2}$ such that $f_{i}(z) \in L_{1}$ for $i=1, \ldots, n$, then for any $i, j \in\{1, \ldots, n\}$ and any $z \in L_{2}$, we have $f_{i}(z) \in L_{1}$ if and only if $f_{j}(z) \in L_{1}$.

There are two possibilities: Either there exist infinitely many $\lambda \in S$ such that $\boldsymbol{a}(\lambda) \in \overline{\mathbb{Q}}$ or not.

Lemma 10.9. If there exist infinitely many $\lambda \in S$ such that $\boldsymbol{a}(\lambda) \in \overline{\mathbb{Q}}$, then $\boldsymbol{a}=\boldsymbol{b}$. In particular, $\operatorname{Prep}(\boldsymbol{a})=\operatorname{Prep}(\boldsymbol{b})$.

Proof. Using Corollary 10.8 we obtain that actually for all $\lambda \in S$ we have that $\boldsymbol{a}(\lambda) \in \overline{\mathbb{Q}}$. So in this case each $\lambda^{\sigma}$ belongs to each $M_{\boldsymbol{a}, v}$ for each place $v$ of the function field $K / \overline{\mathbb{Q}}$ and for each $\sigma \in \operatorname{Gal}(\bar{K} / K)$. (Note that for such $\lambda \in S$ we have that both $f_{\lambda} \in \overline{\mathbb{Q}}[x]$ and $\boldsymbol{a}(\lambda) \in \overline{\mathbb{Q}}$, and also note that $S$ is $\operatorname{Gal}(\bar{K} / K)$-invariant.) Since $M_{\boldsymbol{a}, v}=M_{\boldsymbol{b}, v}$ for each place $v$, we conclude that $\lambda^{\sigma} \in M_{\boldsymbol{b}, v}$ for each $\lambda \in S$, for each $v \in \Omega_{K}$, and for each $\sigma \in \operatorname{Gal}(\bar{K} / K)$. Since $f_{\lambda} \in \overline{\mathbb{Q}}[x]$, we conclude that $\boldsymbol{b}(\lambda) \in \overline{\mathbb{Q}}$ as well. Indeed, otherwise $\left|\boldsymbol{b}(\lambda)^{\sigma}\right|_{v}>1$ for some place $v$ and some Galois morphism $\sigma$, and thus, $\left|f_{\lambda}^{n}\left(\boldsymbol{b}\left(\lambda^{\sigma}\right)\right)\right|_{v} \rightarrow \infty$ as $n \rightarrow \infty$, contradicting the fact that $\lambda^{\sigma} \in M_{\boldsymbol{b}, v}$. Hence, both $\boldsymbol{a}(\lambda) \in \overline{\mathbb{Q}}$ and $\boldsymbol{b}(\lambda) \in \overline{\mathbb{Q}}$ for $\lambda \in S$.

Therefore, applying Lemma 10.6 to the polynomials $c_{0}, \ldots, c_{d-2}, \boldsymbol{a}$, and $\boldsymbol{b}$, we conclude that there exist polynomials $c_{0}^{\prime}, \ldots, c_{d-2}^{\prime}, \boldsymbol{a}^{\prime}, \boldsymbol{b}^{\prime} \in \overline{\mathbb{Q}}[x]$ and $h \in \bar{K}[x]$ such that

$$
\begin{aligned}
c_{i} & =c_{i}^{\prime} \circ h \text { for each } i=0, \ldots, d-2 \text { and } \\
\boldsymbol{a} & =\boldsymbol{a}^{\prime} \circ h \text { and } \boldsymbol{b}=\boldsymbol{b}^{\prime} \circ h .
\end{aligned}
$$

We let $\delta:=h(\lambda)$ and define the family of polynomials

$$
f_{\delta}^{\prime}(x):=x^{d}+\sum_{i=0}^{d-2} c_{i}^{\prime}(\delta) x^{i} .
$$

So we reduced the problem to the case studied in Section 8 for the family of polynomials $f_{\delta}^{\prime} \in \overline{\mathbb{Q}}[x]$ and to the starting points $\boldsymbol{a}^{\prime}, \boldsymbol{b}^{\prime} \in \overline{\mathbb{Q}}[\delta]$. Note that using hypotheses (i) and (ii) from Theorem 2.3 and also relations (10.10) and (10.11), $\boldsymbol{a}^{\prime}(\delta)$ and $\boldsymbol{b}^{\prime}(\delta)$ have the same leading coefficient and the same degree, which is larger than the degrees of the $c_{i}^{\prime}$. So since we know there exist infinitely many $\delta \in \mathbb{C}$ such that $\boldsymbol{a}^{\prime}(\delta)$ and $\boldsymbol{b}^{\prime}(\delta)$ are both preperiodic for $f_{\delta}^{\prime}$, we conclude that $\boldsymbol{a}^{\prime}=\boldsymbol{b}^{\prime}$ as proved in Section 8. Hence, $\boldsymbol{a}=\boldsymbol{b}$, and thus, $\operatorname{Prep}(\boldsymbol{a})=\operatorname{Prep}(\boldsymbol{b})$. 
Lemma 10.12. If finitely many $\lambda \in S$ exist such that $\boldsymbol{a}(\lambda) \in \overline{\mathbb{Q}}$, then $\operatorname{PrepDiff}(\boldsymbol{a}, \boldsymbol{b})$ is finite.

Proof. First, note that there must be at most finitely many $\lambda \in S$ such that $\boldsymbol{b}(\lambda) \in \overline{\mathbb{Q}}$. Otherwise, arguing as in the proof of Lemma 10.9, we would obtain that for all the infinitely many $\lambda \in S$, both $\boldsymbol{a}(\lambda)$ and $\boldsymbol{b}(\lambda)$ are in $\overline{\mathbb{Q}}$, which violates the lemma's hypothesis. So let $T$ be the finite subset of $S$ containing all $\lambda$ such that either $\boldsymbol{a}(\lambda) \in \overline{\mathbb{Q}}$ or $\boldsymbol{b}(\lambda) \in \overline{\mathbb{Q}}$.

Let $\lambda \in(\bar{K} \backslash T) \cap \operatorname{Prep}(\boldsymbol{a})$. If $\lambda \in S$, then by Lemma 10.4 we know that $\lambda \notin \operatorname{Prep}(\boldsymbol{a})$, a contradiction. Therefore, $\lambda \notin S$, so by Proposition 10.3, we have $\lambda \notin \operatorname{PrepDiff}(\boldsymbol{a}, \boldsymbol{b})$. Similarly, if $\lambda \in(\bar{K} \backslash T) \cap \operatorname{Prep}(\boldsymbol{b})$, then $\lambda \notin \operatorname{PrepDiff}(\boldsymbol{a}, \boldsymbol{b})$. Thus, $\operatorname{PrepDiff}(\boldsymbol{a}, \boldsymbol{b})$ is contained in the finite set $T$.

Lemmas 10.9 and 10.12 finish the proof of Proposition 10.5.

Therefore, Proposition 10.5 yields that $\operatorname{Prep}(\boldsymbol{a})$ and $\operatorname{Prep}(\boldsymbol{b})$ differ by at most finitely many elements. Then it follows from Proposition 10.1 that the corresponding complex Mandelbrot sets $M_{a}$ and $M_{b}$ are equal, so we conclude our proof of Theorem 2.3 using Lemma 8.3.

\section{Connections to the dynamical Manin-Mumford conjecture}

We first prove Corollary 2.7, and then we present further connections between our Question 1.3 and the dynamical Manin-Mumford conjecture formulated by Ghioca, Tucker, and Zhang [2011].

Proof of Corollary 2.7. At the expense of replacing $f$ by a conjugate $\delta^{-1} \circ f \circ \delta$ and replacing $\boldsymbol{a}$ and $\boldsymbol{b}$ by $\delta^{-1} \circ \boldsymbol{a}$ and $\delta^{-1} \circ \boldsymbol{b}$, respectively, we may assume $f$ is in normal form. By the hypothesis of Corollary 2.7 , we know that there are infinitely many $\lambda_{n} \in \overline{\mathbb{Q}}$ such that

$$
\lim _{n \rightarrow \infty} \hat{h}_{f}\left(\boldsymbol{a}\left(\lambda_{n}\right)\right)+\hat{h}_{f}\left(\boldsymbol{b}\left(\lambda_{n}\right)\right)=0 .
$$

We let $\boldsymbol{f}:=f_{\lambda}:=f$ be the constant family of polynomials $f$ indexed by $\lambda \in \overline{\mathbb{Q}}$. As before, we let $K$ be the field generated by coefficients of $f, \boldsymbol{a}$, and $\boldsymbol{b}$ and let $h_{\mathbb{M}_{\boldsymbol{a}}}(z)$ and $h_{\mathbb{M}_{b}}(z)$ be the heights of $z \in \bar{K}$ relative to the adelic generalized Mandelbrot sets $\mathbb{M}_{\boldsymbol{a}}:=\prod_{v \in \Omega_{K}} M_{\boldsymbol{a}, v}$ and $\mathbb{M}_{\boldsymbol{b}}$, respectively, as defined in Section 6. So we may apply the equidistribution result from [Baker and Rumely 2010, Theorem 7.52] (see our Theorem 4.3) and conclude that $M_{\boldsymbol{a}, v}=M_{\boldsymbol{b}, v}$ for each place $v \in \Omega_{K}$. Indeed, for each $n \in \mathbb{N}$, we may take $S_{n}$ to be the set of Galois conjugates of $\lambda_{n}$. Clearly $\# S_{n} \rightarrow \infty$ as $n \rightarrow \infty$ (since the points $\lambda_{n}$ are distinct and their heights are bounded because the heights of $\boldsymbol{a}\left(\lambda_{n}\right)$ and $\boldsymbol{b}\left(\lambda_{n}\right)$ are bounded). Finally, $\lim _{n \rightarrow \infty} h_{\mathbb{M}_{a}}\left(S_{n}\right)=$ $\lim _{n \rightarrow \infty} h_{\mathbb{M}_{b}}\left(S_{n}\right)=0$ (by Corollary 6.12), and thus, Theorem 4.3 applies in this case. 
Using that $M_{\boldsymbol{a}, v}=M_{\boldsymbol{b}, v}$ for an archimedean place $v$, the same argument as in the proof of Theorem 2.3 yields that $\boldsymbol{a}=\boldsymbol{b}$ as desired.

Next we discuss the connection between our Question 1.3 and the dynamical Manin-Mumford conjecture [Ghioca et al. 2011, Conjecture 1.4]. First we recall that for a projective variety $X$ and an endomorphism $\Phi$ of $X$, we say that $\Phi$ is polarizable if there exists an integer $d>1$ and there exists an ample line bundle $\mathscr{L}$ on $X$ such that $\Phi^{*}(\mathscr{L})=\mathscr{L}^{\otimes d}$.

Conjecture 11.1 (Ghioca, Tucker, Zhang). Let $X$ be a projective variety, define $\varphi: X \rightarrow X$ to be a polarizable endomorphism defined over $\mathbb{C}$, and let $Y$ be a subvariety of $X$ that has no component included into the singular part of $X$. Then $Y$ is preperiodic under $\varphi$ if and only if there exists a Zariski dense subset of smooth points $x \in Y \cap \operatorname{Prep}_{\varphi}(X)$ such that the tangent subspace of $Y$ at $x$ is preperiodic under the induced action of $\varphi$ on the Grassmannian $\operatorname{Gr}_{\operatorname{dim}(Y)}\left(T_{X, x}\right)$. (Here $T_{X, x}$ denotes the tangent space of $X$ at the point $x$.)

Ghioca, Tucker, and Zhang [2011] prove that Conjecture 11.1 holds whenever $\Phi$ is a polarizable algebraic group endomorphism of the abelian variety $X$ and also when $X=\mathbb{P}^{1} \times \mathbb{P}^{1}, Y$ is a line, and $\Phi(x, y)=(f(x), g(y))$ for any rational maps $f$ and $g$. We claim that a positive answer to Question 1.3 yields the following special case of Conjecture 11.1 that is not covered by the results from [Ghioca et al. 2011]. Note that we do not need the condition on preperiodicity of tangent spaces in the Grassmannian, only an infinite family of preperiodic points; hence, what one would obtain here is really a special case of Zhang's original dynamical Manin-Mumford conjecture (which did not require the extra hypothesis on tangent spaces).

Proposition 11.2. If Question 1.3 holds in the affirmative, then for any endomorphism $\Phi$ of $\mathbb{P}^{1} \times \mathbb{P}^{1}$ given by $\Phi(x, y):=(f(x), f(y))$ for some rational map $f \in \mathbb{C}(x)$ of degree at least 2 , a curve $Y \subset \mathbb{P}^{1} \times \mathbb{P}^{1}$ will contain infinitely many preperiodic points if and only if $Y$ is preperiodic under $\Phi$. In particular, Question 1.3 implies Conjecture 11.1 for such $Y$ and $\Phi$.

Proof. Let $Y \subset \mathbb{P}^{1} \times \mathbb{P}^{1}$ be a curve containing infinitely many points $(x, y)$ such that both $x$ and $y$ are preperiodic for $f$. Furthermore, we may assume $Y$ projects dominantly on each coordinate of $\mathbb{P}^{1} \times \mathbb{P}^{1}$ since otherwise it is immediate to conclude that $Y$ contains infinitely many preperiodic points for $\Phi$ if and only if $Y=\{c\} \times \mathbb{P}^{1}$ or $Y=\mathbb{P}^{1} \times\{c\}$, where $c$ is a preperiodic point for $f$.

We let $f=f_{\lambda}:=f$ be the constant family of rational functions (equal to $f$ ) indexed by all points $\lambda \in Y$ and let $K$ be the function field of $Y$. Let $(\boldsymbol{a}, \boldsymbol{b}) \in$ $\mathbb{P}^{1}(K) \times \mathbb{P}^{1}(K)$ be a generic point for $Y$. By our assumption, there exist infinitely many $\lambda \in Y$ such that both $\boldsymbol{a}(\lambda)$ and $\boldsymbol{b}(\lambda)$ are preperiodic for $f_{\lambda}=f$. Since $Y$ projects dominantly on each coordinate of $\mathbb{P}^{1} \times \mathbb{P}^{1}$, we get that neither $\boldsymbol{a}$ nor $\boldsymbol{b}$ 
is preperiodic under the action of $f$. (Otherwise, $\boldsymbol{a}$ or $\boldsymbol{b}$ would be constant.) So assuming the answer to Question 1.3 is "yes", we obtain that the curve $Y(\mathbb{C})=$ $\{(\boldsymbol{a}(\lambda), \boldsymbol{b}(\lambda)): \lambda \in Y\} \subset \mathbb{P}_{K}^{1} \times_{K} \mathbb{P}_{K}^{1}$ lies on a preperiodic proper subvariety $Z$ of $\mathbb{P}^{1} \times \mathbb{P}^{1}$ defined over a finite extension of $K$. More precisely, we get that $Z=Y \otimes_{\mathbb{C}} K$, so $Y$ must be itself preperiodic under the action of $(f, f)$ on $\mathbb{P}^{1} \times \mathbb{P}^{1}$.

Conversely, suppose that $Y$ is preperiodic under $\Phi$. Then some iterate of $Y$ contains a dense set of periodic points by [Fakhruddin 2003], so $Y$ contains an infinite set of preperiodic points.

Remarks 11.3. (a) In the proof of Proposition 11.2, we did not use the full strength of the hypothesis from Conjecture 11.1. Instead we used the weaker hypothesis of [Zhang 1995, Conjecture 2.5] or [Zhang 2006, Conjecture 1.2.1, Conjecture 4.1.7] (which was the original formulation of the dynamical Manin-Mumford conjecture). This is not surprising since for curves contained in $\mathbb{P}^{1} \times \mathbb{P}^{1}$, the only counterexamples to the original formulation of the dynamical Manin-Mumford conjecture are expected to occur when $\Phi:=(f, g)$ for two distinct Lattès maps.

(b) Finally, we note that a positive answer to Conjecture 11.1 does not yield a positive answer to Question 1.3. Instead, Question 1.3 goes in a different direction that is likely to shed more light on the dynamical Manin-Mumford conjecture especially in the case when $Y$ is a curve in Conjecture 11.1.

\section{Acknowledgments}

Hsia acknowledges the support from the 2010-2011 France-Taiwan Orchid Program, which enabled him to attend the Summer School on Berkovich spaces held at Institut de Mathématiques de Jussieu, where this project was initiated in the summer of 2010. We thank Matthew Baker, Laura DeMarco, and Joseph Silverman for several useful conversations and also thank the referee for comments.

\section{References}

[Baker 2009] M. Baker, "A finiteness theorem for canonical heights attached to rational maps over function fields”, J. Reine Angew. Math. 626 (2009), 205-233. MR 2011c:14075 Zbl 1187.37133

[Baker and DeMarco 2011] M. Baker and L. DeMarco, "Preperiodic points and unlikely intersections", Duke Math. J. 159:1 (2011), 1-29. MR 2012h:37170 Zbl 1242.37062

[Baker and Hsia 2005] M. H. Baker and L.-C. Hsia, "Canonical heights, transfinite diameters, and polynomial dynamics”, J. Reine Angew. Math. 585 (2005), 61-92. MR 2006i:11071 Zbl 1071.11040

[Baker and Rumely 2010] M. Baker and R. Rumely, Potential theory and dynamics on the Berkovich projective line, Mathematical Surveys and Monographs 159, American Mathematical Society, Providence, RI, 2010. MR 2012d:37213 Zbl 1196.14002

[Beardon 1991] A. F. Beardon, Iteration of rational functions, Graduate Texts in Mathematics 132, Springer, New York, 1991. MR 92j:30026 Zbl 0742.30002 
[Benedetto 2005] R. L. Benedetto, "Heights and preperiodic points of polynomials over function fields”, Int. Math. Res. Not. 2005:62 (2005), 3855-3866. MR 2006m:11095 Zbl 1114.14018

[Berkovich 1990] V. G. Berkovich, Spectral theory and analytic geometry over non-Archimedean fields, Mathematical Surveys and Monographs 33, American Mathematical Society, Providence, RI, 1990. MR 91k:32038 Zbl 0715.14013

[Bertrand 2011] D. Bertrand, "Special points and Poincaré bi-extensions, with an Appendix by Bas Edixhoven”, preprint, 2011. arXiv 1104.5178

[Bombieri and Gubler 2006] E. Bombieri and W. Gubler, Heights in Diophantine geometry, New Mathematical Monographs 4, Cambridge University Press, 2006. MR 2007a:11092 Zbl 1115.11034

[Bombieri et al. 1999] E. Bombieri, D. Masser, and U. Zannier, "Intersecting a curve with algebraic subgroups of multiplicative groups", Internat. Math. Res. Notices 20 (1999), 1119-1140. MR 2001c:11081 Zbl 0938.11031

[Branner and Hubbard 1988] B. Branner and J. H. Hubbard, "The iteration of cubic polynomials. I. The global topology of parameter space”, Acta Math. 160:3-4 (1988), 143-206. MR 90d:30073 Zbl 0668.30008

[Call and Silverman 1993] G. S. Call and J. H. Silverman, "Canonical heights on varieties with morphisms”, Compositio Math. 89:2 (1993), 163-205. MR 95d:11077 Zbl 0826.14015

[Carleson and Gamelin 1993] L. Carleson and T. W. Gamelin, Complex dynamics, Springer, New York, 1993. MR 94h:30033 Zbl 0782.30022

[Fakhruddin 2003] N. Fakhruddin, "Questions on self maps of algebraic varieties", J. Ramanujan Math. Soc. 18:2 (2003), 109-122. MR 2004f:14038 Zbl 1053.14025

[Ghioca and Tucker 2008] D. Ghioca and T. J. Tucker, "Equidistribution and integral points for Drinfeld modules", Trans. Amer. Math. Soc. 360:9 (2008), 4863-4887. MR 2009c:11095 Zbl 1178.11046

[Ghioca et al. 2011] D. Ghioca, T. J. Tucker, and S. Zhang, “Towards a dynamical Manin-Mumford conjecture”, Int. Math. Res. Not. 2011:22 (2011), 5109-5122. MR 2012i:37142 Zbl 05988299

[Habegger 2009] P. Habegger, "Intersecting subvarieties of abelian varieties with algebraic subgroups of complementary dimension”, Invent. Math. 176:2 (2009), 405-447. MR 2010f:11109 Zbl 1176.14008

[Hsia 2008] L.-C. Hsia, "On the reduction of a non-torsion point of a Drinfeld module", J. Number Theory 128:6 (2008), 1458-1484. MR 2009f:11065 Zbl 1144.11046

[Lang 1983] S. Lang, Fundamentals of Diophantine geometry, Springer, New York, 1983. MR 85j: 11005 Zbl 0528.14013

[Masser and Zannier 2010] D. Masser and U. Zannier, "Torsion anomalous points and families of elliptic curves", Amer. J. Math. 132:6 (2010), 1677-1691. MR 2012d:11133 Zbl 1225.11078

[Masser and Zannier 2012] D. Masser and U. Zannier, "Torsion points on families of squares of elliptic curves”, Math. Ann. 352:2 (2012), 453-484. MR 2012k:11076 Zbl 06006368

[Pink 2005] R. Pink, "A combination of the conjectures of Mordell-Lang and André-Oort", pp. 251-282 in Geometric methods in algebra and number theory, edited by F. Bogomolov and Y. Tschinkel, Progr. Math. 235, Birkhäuser, Boston, MA, 2005. MR 2006f:11068 Zbl 1200.11041

[Ransford 1995] T. Ransford, Potential theory in the complex plane, London Mathematical Society Student Texts 28, Cambridge University Press, 1995. MR 96e:31001 Zbl 0828.31001

[Raynaud 1983a] M. Raynaud, "Courbes sur une variété abélienne et points de torsion", Invent. Math. 71:1 (1983), 207-233. MR 84c:14021 Zbl 0564.14020

[Raynaud 1983b] M. Raynaud, "Sous-variétés d'une variété abélienne et points de torsion", pp. 327352 in Arithmetic and geometry, vol. I, edited by M. Artin and J. Tate, Progr. Math. 35, Birkhäuser, Boston, MA, 1983. MR 85k:14022 Zbl 0581.14031 
[Shafarevich 1994] I. R. Shafarevich, Basic algebraic geometry, I, 2nd ed., Springer, Berlin, 1994. MR 95m:14001 Zbl 0797.14001

[Silverman 1994a] J. H. Silverman, "Variation of the canonical height on elliptic surfaces, II: Local analyticity properties”, J. Number Theory 48:3 (1994), 291-329. MR 95h:11054 Zbl 0807.14019

[Silverman 1994b] J. H. Silverman, "Variation of the canonical height on elliptic surfaces, III: Global boundedness properties", J. Number Theory 48:3 (1994), 330-352. MR 95h:11055 Zbl 0807.14020

[Zhang 1995] S. Zhang, "Small points and adelic metrics", J. Algebraic Geom. 4:2 (1995), 281-300. MR 96e:14025 Zbl 0861.14019

[Zhang 2006] S.-W. Zhang, "Distributions in algebraic dynamics", pp. 381-430 in Surveys in differential geometry, vol. 10, edited by S. T. Yau, Surv. Differ. Geom. 10, Int. Press, Somerville, MA, 2006. MR 2009k:32016 Zbl 1207.37057

Communicated by Bjorn Poonen

Received 2011-09-21 Revised 2012-03-25 Accepted 2012-03-29

dghioca@math.ubc.ca Department of Mathematics, University of British Columbia, Vancouver, BC V6T 1Z2, Canada

hsia@math.ntnu.edu.tw Department of Mathematics, National Taiwan Normal University, Taipei, Taiwan

ttucker@math.rochester.edu Department of Mathematics, University of Rochester, Rochester, NY 14627, United States 


\section{Algebra \& Number Theory}

msp.org/ant

\section{EDITORS}

MANAGING EDITOR

Bjorn Poonen

Massachusetts Institute of Technology

Cambridge, USA

\author{
EDITORIAL BOARD CHAIR \\ David Eisenbud \\ University of California \\ Berkeley, USA
}

\section{BOARD OF EDITORS}

Georgia Benkart

Dave Benson

Richard E. Borcherds

John H. Coates

J-L. Colliot-Thélène

Brian D. Conrad

Hélène Esnault

Hubert Flenner

Edward Frenkel

Andrew Granville

Joseph Gubeladze

Ehud Hrushovski

Craig Huneke

Mikhail Kapranov

Yujiro Kawamata

János Kollár

Yuri Manin

Barry Mazur

Philippe Michel
University of Wisconsin, Madison, USA

University of Aberdeen, Scotland

University of California, Berkeley, USA

University of Cambridge, UK

CNRS, Université Paris-Sud, France

University of Michigan, USA

Freie Universität Berlin, Germany

Ruhr-Universität, Germany

University of California, Berkeley, USA

Université de Montréal, Canada

San Francisco State University, USA

Hebrew University, Israel

University of Virginia, USA

Yale University, USA

University of Tokyo, Japan

Princeton University, USA

Northwestern University, USA

Harvard University, USA

École Polytechnique Fédérale de Lausanne
Susan Montgomery

Shigefumi Mori

Raman Parimala

Jonathan Pila

Victor Reiner

Karl Rubin

Peter Sarnak

Joseph H. Silverman

Michael Singer

Vasudevan Srinivas

J. Toby Stafford

Bernd Sturmfels

Richard Taylor

Ravi Vakil

Michel van den Bergh

Marie-France Vignéras

Kei-Ichi Watanabe

Efim Zelmanov
University of Southern California, USA

RIMS, Kyoto University, Japan

Emory University, USA

University of Oxford, UK

University of Minnesota, USA

University of California, Irvine, USA

Princeton University, USA

Brown University, USA

North Carolina State University, USA

Tata Inst. of Fund. Research, India

University of Michigan, USA

University of California, Berkeley, USA

Harvard University, USA

Stanford University, USA

Hasselt University, Belgium

Université Paris VII, France

Nihon University, Japan

University of California, San Diego, USA

\section{PRODUCTION}

production@msp.org

Silvio Levy, Scientific Editor

See inside back cover or msp.org/ant for submission instructions.

The subscription price for 2013 is US \$200/year for the electronic version, and \$350/year ( $\$ 40$, if shipping outside the US) for print and electronic. Subscriptions, requests for back issues and changes of subscribers address should be sent to MSP.

Algebra \& Number Theory (ISSN 1944-7833 electronic, 1937-0652 printed) at Mathematical Sciences Publishers, 798 Evans Hall \#3840, c/o University of California, Berkeley, CA 94720-3840 is published continuously online. Periodical rate postage paid at Berkeley, CA 94704, and additional mailing offices.

ANT peer review and production are managed by EditFLOW ${ }^{\circledR}$ from Mathematical Sciences Publishers.

\section{PUBLISHED BY}

- mathematical sciences publishers

nonprofit scientific publishing

http://msp.org/

(C) 2013 Mathematical Sciences Publishers 


\section{Algebra \& Number Theory}

Volume $7 \quad$ No. $3 \quad 2013$

Ekedahl-Oort strata of hyperelliptic curves in characteristic 2

ARSEN ELKIN and RACHEL PRIES

Cycle classes and the syntomic regulator

Bruno Chiarellotto, Alice Ciccioni and Nicola Mazzari

Zeros of real irreducible characters of finite groups

SELENA MARINELli and PHAM HUU TIEP

The biHecke monoid of a finite Coxeter group and its representations

FLORENT HIVERT, ANNE SCHILLING and NICOLAS THIÉRY

Shuffle algebras, homology, and consecutive pattern avoidance

VLADIMIR DOTSENKO and ANTON KHOROSHKIN

701

Preperiodic points for families of polynomials

Dragos Ghioca, Liang-Chung Hsia and Thomas J. Tucker

$F$-blowups of normal surface singularities

NobUo HaRa, TADAKaZU SAWADA and TAKEHIKo YASUdA 